\title{
Quantifying community-wide antimicrobials usage via wastewater-based epidemiology
}

\author{
Elizabeth Holton ${ }^{1}$, Natalie Sims ${ }^{1}$, Kishore Jagadeesan ${ }^{1}$, Richard Standerwick², \\ Barbara Kasprzyk-Hordern ${ }^{*}$ \\ ${ }^{1}$ University of Bath, Department of Chemistry, Bath, BA2 7AY, UK \\ ${ }^{2}$ Wessex Water, Claverton Down Rd, Bath, BA2 7WW, UK \\ *Corresponding author. Email address: b.kasprzyk-hordern@bath.ac.uk
}

\begin{abstract}
Increasing usage of antimicrobials is a significant contributor to the emergence and dissemination of antimicrobial resistance. Wastewater-based epidemiology is a useful tool for evaluating public health, via the monitoring of chemical and biological markers in wastewater influent, such as antibiotics. Chemical analyses are used to determine sampled drug concentrations; measured daily flows then enable quantitation of analyte mass/day; and population estimates are utilised to calculate mass/day/1000inhabitants. These data allow for effective evaluations of drug presence and temporal trends, but do not fully represent the total quantity of drugs being consumed, i.e., human intake. Factors such as drug metabolism and physiochemical stability significantly decrease the quantity of parent drug that reaches wastewater treatment plants, leading to potential underestimations of community usage. A case study of 16 antimicrobials, and their metabolites was conducted in this study: including sulfonamides, trimethoprim, metronidazole, quinolones, nitrofurantoin, cyclines, and antiretrovirals. Correction factors ( $\mathrm{CFs}$ ) for human drug excretion, for various metabolite forms, were determined via a systematic literature review of pharmacokinetic research. Analyte stability was examined over a $24 \mathrm{~h}$ study. The estimation of community-wide drug intake was evaluated using the associated catchment prescription data. Overall, antimicrobials excreted in an unchanged form were often observed to over-estimate daily intake. This could be attributed to biotransformation, e.g., via glucuronide cleavage, or direct disposal of unused drugs. Acetylsulfonamides, trimethoprim, hydroxy-metronidazole, clarithromycin, ciprofloxacin, ofloxacin, tetracycline, and oxytetracycline generally performed well in the estimation of drug intake, relative to prescription records. The low prevalence of quinolone and trimethoprim metabolites, and the low stability of nitrofurantoin, limited the ability to evaluate these metabolites and their respective CFs. CFs established in the systematic literature review could not be validated for some metabolites in the case study due to lack of available prescription data (lamivudine, emtricitabine); an inability to quantify biomarkers (nitrofurantoin, doxycycline); being excreted at too low levels (hydroxy-trimethoprim, ofloxacin-N-oxide, desethylene-ciprofloxacin); or insufficient pharmacokinetic literature sources (the nitrofurantoin metabolite, NPAHD). Further work is currently underway to apply established CFs in other catchment with higher prevalence of antimicrobials usage.
\end{abstract}

Keywords: antibiotic/antimicrobial, metabolism, excretion, prescription, wastewater-based epidemiology (WBE)

\section{Introduction}

Antimicrobial agents have revolutionised medical care in the twentieth century as they have contributed to a dramatic reduction in mortality from infectious disease. Antimicrobial resistance (AMR) is to large extent a consequence of extensive and uncontrolled antimicrobial use. There are several reports emphasising the urgent need to introduce effective strategies to control and prevent AMR [1-3]. Among the initiatives introduced to combat the problem of AMR are surveillance and increased awareness of AMR; appropriate use of antimicrobials; effective infection control; improved vaccination; and relevant policies for, and regulations of, antimicrobials and AMR. Unfortunately, there is little understanding regarding spatiotemporal antimicrobials usage, due to lack of robust surveillance tools.

Wastewater-Based Epidemiology (WBE) provides an attractive solution. Wastewater is a complex mixture of substances including a wide range of human excretion products, resulting from public exposure to stressors (e.g., toxicants and infectious agents) and physiological processes (e.g., specific disease-linked proteins, genes, and metabolites). Endogenous and exogenous residues, both environment and human derived, are continuously pooled by the sewerage system serving a community. Quantitative measurement of these chemical, biological, and physical stressors can provide evidence of exposure to a surveyed population, and profile the associated effects anonymously, at low cost, and in near-real time. Wastewater (WW) can therefore be considered as a diagnostic medium for the health status of a community [4].

WBE is often used to estimate illicit drug use trends [5, 6], but has also been applied to estimate public exposure to alcohol [7, 8]; tobacco [9]; counterfeit medicines [10, 11]; as well as levels of stress biomarkers, such as isoprostanes $[12,13]$. WBE has revolutionised population health studies, especially in the context of the COVID 
pandemic [14-17]. There are, however, only a very few reports focussing on public exposure to chemicals such as pesticides [18-20]), industrial chemicals [21, 22], or antibiotics [23] [24]. This is mainly due to a limited understanding of drug-specific human metabolism and difficulties in calculating metabolism correction factors from literature resources.

This manuscript presents the strong potential of using WBE as an epidemiology tool aimed to estimate community-wide intake of antimicrobials. Its main objectives are:

1. Systematic review of literature data on human metabolism and establishment of a robust CF (metabolic transformation Correction Factor) estimation workflows

2. Model calibration using Southwest England (Bristol-Avon catchment) and per postcode antimicrobial prescription model

3. Estimation of antimicrobial intake in Southwest England

\section{Materials and methods}

2.1. Materials and target drugs

Analytical standards and deuterated (stable isotope-labelled) standards were purchased from Sigma-Aldrich (Gillingham, UK), TRC (Toronto, Canada), LGC (Middlesex, UK), or MCE (Cambridge, UK); the list of which is collated in SI tab.1 Methanol, $\mathrm{MeOH}$, was HPLC grade (Sigma-Aldrich). Water, $\mathrm{H}_{2} \mathrm{O}$, was of $18.2 \mathrm{M} \Omega$ quality (Elga, Marlow, UK). Glassware was deactivated using 5\% dimethyldichlorosilane (DMDCS) in toluene (SigmaAldrich) to mitigate the loss of basic chemicals onto $-\mathrm{OH}$ sites present on glass surfaces. This consisted of rinsing once with DMDCS, twice with toluene and three times with $\mathrm{MeOH}$. The mobile phase buffer was formic acid $(>95 \%, \mathrm{HCOOH})$, purchased from Sigma-Aldrich. Oasis HLB (60 mg, $3 \mathrm{~mL})$ SPE cartridges, polypropylene LC vials, and Whatman GF/F $0.7 \mu \mathrm{m}$ filters were purchased from Waters (Manchester, UK).

Sixteen drug targets, including 29 metabolites, were selected. This list includes several antimicrobial classes, resulting in a broad range of physiochemical and pharmacokinetic behaviour (Table1).

Table 1 Drug targets and abbreviations

\begin{tabular}{|c|c|c|c|c|}
\hline Drug class & Parent drug & Abbrev. & Drug metabolite & Abbrev. \\
\hline \multirow[t]{4}{*}{ Sulfonamide } & Sulfamethoxazole & SMX & Acetyl-sulfamethoxazole & $\operatorname{aSMX}$ \\
\hline & Sulfadiazine & SDZ & Acetyl-sulfadiazine & aSDZ \\
\hline & Sulfasalazine & SLZ & Sulfapyridine & SPY \\
\hline & & & Acetyl-sulfapyridine & aSPY \\
\hline Trimethoprim & Trimethoprim & TMP & Hydroxy-trimethoprim & hTMP \\
\hline Nitrofuran & Nitrofurantoin & NIT & $\begin{array}{l}\text { 1-(2-nitrobenzylidenamino)-2,4- } \\
\text { imidazolidinedione }\end{array}$ & NPAHD \\
\hline \multirow[t]{4}{*}{ Quinolone } & Ciprofloxacin & CIP & Desethylene-ciprofloxacin & deCIP \\
\hline & Ofloxacin/ Levofloxacin & OFX & Desmethyl-ofloxacin & dmOFX \\
\hline & & & Ofloxacin $\mathrm{N}$-oxide & OFXo \\
\hline & Norfloxacin & NOR & Hydroxy-norfloxacin & hNOR \\
\hline Azole & Metronidazole & MTZ & Hydroxy-metronidazole & hMTZ \\
\hline Macrolide & Clarithromycin & CLR & Desmethyl-clarithromycin & $\mathrm{dmCLR}$ \\
\hline Lincomycin & Clindamycin & $\mathrm{CLI}$ & Desmethyl-clindamycin & $\mathrm{dmCLI}$ \\
\hline \multirow[t]{2}{*}{$\begin{array}{l}\text { Antiretrovirals } \\
\text { (ARVs) }\end{array}$} & Emtricitabine & FTC & & \\
\hline & Lamivudine & 3TC & & \\
\hline \multirow[t]{3}{*}{ Cycline } & Tetracycline & TET & & \\
\hline & Doxycycline & DOX & & \\
\hline & Oxytetracycline & OTC & & \\
\hline
\end{tabular}


Wastewater samples were obtained during two sampling campaigns during winter and summer months of 2019 (Jan/Feb and Jun/Jul, respectively). The wastewater treatment plant (WWTP), located in the Southwest of England, receives wastewater from a city with an estimated 120,113 inhabitants (2019), and a $1.2 \%$ wastewater contribution from industry (incl. commercial PE and trade effluent s seen in Table 2). Raw wastewater samples were collected as flow proportional $24 \mathrm{~h}$ composites (ISCO 3700 autosampler), with average sub-sample collection frequencies of approximately 15 minutes. The autosampler was packed with ice to maintain an approximate temperature of $4^{\circ} \mathrm{C}$ during collection, in order to limit biological activity [25]. All samples were transported on ice to the laboratory, spiked with the internal standards, and stored at $-18^{\circ} \mathrm{C}$ until sample preparation and analysis.

\subsection{Sample preparation and analyte quantitation}

Sample preparation and analysis were performed using a method previously described by Holton et al. [26]. Briefly, selected analytes were extracted from wastewater and concentrated using Solid-Phase Extraction (SPE). Wastewater samples $(50 \mathrm{~mL})$ were spiked with $50 \mathrm{ng}$ of each internal standard $\left(50 \mu \mathrm{L}\right.$ of $1 \mu \mathrm{g} \mathrm{mL}^{-1}$, filtered using a GF/F filter paper (Whatman, $0.7 \mu \mathrm{m})$ and loaded under vacuum onto conditioned Oasis HLB cartridges $(2 \mathrm{~mL}$ $\mathrm{MeOH}$, followed by $2 \mathrm{~mL} \mathrm{H} 2 \mathrm{O}$ ) at approx. $5 \mathrm{~mL} \mathrm{~min}^{-1}$. Cartridges were dried under vacuum, sealed with parafilm, and stored at $-20{ }^{\circ} \mathrm{C}$ until elution with $4 \mathrm{~mL} \mathrm{MeOH}$ to deactivated glass vials followed by drying under nitrogen, at $40{ }^{\circ} \mathrm{C}$ and re-suspension $(500 \mu \mathrm{L}$ 80:20 H2O:MeOH) into LC vials (Waters). Reverse-phase liquid chromatography (Waters, ACQUITY UPLC ${ }^{\mathrm{TM}}$ ) coupled with tandem mass spectrometry (Xevo TQD-ESI+) was utilised, via multiple reaction monitoring acquisition. Chromatographic separation of each $20 \mu \mathrm{L}$ sample was achieved over a 19 min gradient elution using $\mathrm{H}_{2} \mathrm{O}: \mathrm{MeOH}$ (95:5, with $0.1 \%$ formic acid), and methanol (100\%) as mobile phases. Weighted calibration curves were produced in replicate using stable isotope labelled internal standards. Accuracy, precision, analyte recovery, and method limits are outlined in SI tab.2 and SI fig.8.

\subsection{Systematic database searching protocol for drug pharmacokinetics data}

To establish human drug intake, the percentage excretions of the parent/unchanged drug and other drug metabolites were required. These data were extracted from pharmacokinetic research papers, with and without the use of a systematic database searching protocol.

The protocol was designed around the Web of Science advanced search platform. The Web of Science 'Core Collection' database does not contain literature publisher prior to 1970, which was unsuitable for antibiotic research. Therefore, the option for 'All Databases' was selected, spanning from 1864 to present. Searches were performed and combined to produce an initial pool of pharmacokinetic results, from which drug-specific papers were extracted. The search terms used are outlined in SI tab.5.

The Boolean operator 'NEAR' was used to refine the result pool. This choice was weighed against the possibility of missing papers without abstracts on file in the metadata, and therefore less likely to meet the search criteria. To compensate, a broader search term was included which prioritised the terms 'metaboli*', 'parent', 'unchanged', and 'pharmacokinetic*'. Where possible, alternative spellings were accounted for using the asterisk (*) wildcard. For synonyms, 'sul*onamide' returns hits for both 'sulfonamide' and 'sulphonamide'. This was also adopted for suffixes; such as, using 'excret*,' for 'excrete', 'excretes', 'excreted', 'excretion', etc. The question mark (?) wildcard was used to replace a single character; for example, 'wom?n' returns hits for both 'woman' and 'women'. Alternative drug names were also considered, such as sulfasalazine, otherwise known as salicylazosulfapyridine, or salazosulfapyridine, or SASP.

2.5. Antimicrobial intake calculation

The daily quantity of each antimicrobial was estimated from wastewater samples as well as prescription records. These were then normalised for the specific catchment region using population estimates (PE). PE was determined via two methods: NHS registered patients (PE-NHS) and WWTP water utility estimates (PE-WW). Terminology used is gathered in Table 2 .

Table 2 Terminology used

\begin{tabular}{ll}
\hline Acronym & Description \\
\hline WW & Wastewater \\
WWTP & Wastewater treatment plant \\
CF & Correction factor (using metabolite excretion percentage and molecular weight) \\
PE & Population estimate \\
PE-WW & Population estimate, derived from WWTP
\end{tabular}




\begin{tabular}{ll} 
PE-NHS & Population estimate, derived from registered NHS patients \\
DL & Daily load, derived from WW concentration and daily flow rate \\
PNDL & Population normalised daily load, derived from DL and PE-WW \\
DI & Daily intake, derived from PNDL and analyte CF \\
PPPD & Per postcode prescription data \\
PNDP & Population normalised daily prescription, derived from prescribed mass loads and PE-WW \\
Ratio-WW & Ratio of WW loads between two analytes (PNDL or DI) \\
Ratio-NHS & Ratio of PNDPs between two drugs (e.g., SMX/TMP) \\
\hline
\end{tabular}

\subsubsection{Population equivalent estimation}

The population estimate obtained from the WWTP (PE-WW) was based on water utility estimates (Table 3) [27]. The resident PE was determined via the number of properties and average occupancy, including adjustments for care homes, schools, colleges, and universities. Average household size is calculated per district.

The resident population includes care homes, schools, prisons, and military bases, whereas the non-resident population includes overnight tourism. Commercial waste was estimated to contribute $60 \mathrm{~g}$ BOD (biochemical oxygen demand) per capita, per day. Tankered waste, including septic and industrial waste, was estimated from the chemical oxygen demand (COD), per unit volume; via an assumption of $120 \mathrm{~g}$ COD per capita per day. The population estimate established from NHS surgery registration (PE-NHS), was determined for the specific WWTP catchment region. NHS Digital was used to obtain GP surgery postcodes and distribution of registered individuals. A data processing tool, PrAna, was used to collate and process these data in association with catchment maps provided by the WWTP [28].

Load and intake calculations were all performed using the population estimate obtained from the WWTP (PEWW). The confidence in this value was validated by the concordance to the corresponding number of patients registered (PE-NHS).

Table 3 Population equivalents (PE) for the WWTP catchment (2019)

\begin{tabular}{ll}
\hline City/Town statistics & Estimate \\
\hline Domestic-Billed properties & 45,274 \\
Average household size & 2.25 \\
Resident population estimate & 101,866 \\
Adjustment for Care Homes & 1,411 \\
Adjustment for Universities & 5,800 \\
Adjustment for Schools/Colleges & 800 \\
Non-Resident population estimate & 7,250 \\
Commercial population estimate & 2,006 \\
Trade Effluent population estimate & 980 \\
Tankered Waste population estimate & 0 \\
\hline Total PE served by WWTP (PE-WW 2019) & $\mathbf{1 2 0 , 1 1 3}$ \\
Patients registered (PE-NHS 2019) & $\mathbf{1 1 8 , 6 8 6} \pm \mathbf{1 7 5 . 5} *$ \\
CV \% & 0.85 \\
\hline
\end{tabular}

* Average of the number patients registered for four months (Jan, Feb, Jun, and Jul 2019)

\subsubsection{Daily mass load (DL), population normalised mass load (PNDL) and daily intake (DI) from WBE data}

Analyte daily mass loads $\left(D L, \mathrm{mg} \mathrm{day}^{-1}\right)$ were calculated by multiplying the total analyte (parent or metabolite) concentration $\left(\mathrm{mg} \mathrm{L}^{-1}\right)$, by daily wastewater flow rates $\left(\mathrm{L} \mathrm{day}^{-1}\right)$, Equ.1. Total analyte concentrations, obtained from a $24 \mathrm{~h}$ composite raw wastewater sample, were calculated whilst accounting for both liquid and SPM fractions, by spiking with ISTD prior to filtration.

Equation 1: $\quad D L_{\text {Analyte }}\left[m g d a y^{-1}\right]=C_{\text {Analyte }} V$

In Equ.1, $C_{\text {Analyte }}$ is the total concentration of analyte $\left(\mathrm{mg} \mathrm{L}^{-1}\right)$ in influent wastewater, and $V$ is the volume of wastewater received by the WWTP per day $\left(\mathrm{L} \mathrm{day}^{-1}\right)$. The mass loads of analytes were then normalized for the 
number of people served by each WWTP to give population normalised daily loads ( $P N D L, \mathrm{mg} \mathrm{day}^{-1} 1000$ inhabitants ${ }^{-1}$ ). PNDL enables comparisons between different WWTPs.

$$
\text { Equation 2: } \quad P N D L_{\text {Analyte }}\left[m g \text { day }^{-1} 1000 \operatorname{inh}^{-1}\right]=D L_{\text {Analyte }}\left(\frac{1000}{P E_{W W}}\right)
$$

In Equ.2, $P E-W W$ is the population size served by the WWTP. Population-wide antimicrobial daily intake (DI, $\mathrm{mg} \mathrm{day}^{-1} 1000 \mathrm{inh}^{-1}$ ) was calculated using the following equation:

Equation 3: $\quad$ DI $I_{\text {Antimicrobial }}\left[\mathrm{mg} \mathrm{day}^{-1} 1000 \mathrm{inh}^{-1}\right]=P N D L_{\text {Analyte }} C F_{\text {Analyte }}$

In Equ.3, $C F$ is the correction factor; calculated using equation 4. All correction factors, and associated literature references, are outlined in Table 5 and SI tab.6.

Equation 4:

$$
C F=\frac{\frac{M W_{\text {Antimicrobial }}}{M W_{\text {Analyte }}}}{\text { Excretion }_{\text {Analyte }}} * 100
$$

$M W_{\text {Antimicrobial }}$ and $M W_{\text {Analyte }}$ are the molecular weights of the analyte (either parent compound or metabolite) and antimicrobial (parent), respectively. This MW function is included to correct for the metabolic stoichiometry, when working with quantities by mass. Excretion Analyte $_{(\%)}$ is the percentage of analyte excreted in urine and faeces, as determined via pharmacokinetic literature review.

$$
\begin{array}{ll}
\text { Equation 5: } & \text { Ratio }_{W W}=P N D L_{\text {Antimicrobial }} / P N D L_{\text {Metabolite }} \\
\text { or } & \text { Ratio }_{W W}=D I_{\text {Antimicrobial }} / D I_{\text {Metabolite }}
\end{array}
$$

Analyte ratios in wastewater (Ratio-WW) are calculated to observe the relationship between parent and metabolite forms, and how the ratio changes after applying analyte CFs. They are also effective in identifying events of drug disposal.

\subsubsection{Per Postcode Prescription Data (PPPD) and population normalised daily prescription (PNDP)}

The total quantity of prescribed antimicrobials $(\mathrm{kg} / \mathrm{month})$ was calculated for the catchment region, using the $\mathrm{R}$ package, PrAna [28]. Briefly, postcodes corresponding to each catchment region were identified using WWTP catchment maps provided by Wessex Water. Based on postcode, the general practices (GPs) in the regions were identified. The total mass of individual active pharmaceutical ingredient could then be calculated for a specified catchment area and time period. This was achieved using PrAna, utilising publicly available prescription data published by NHS Digital (https://digital.nhs.uk/).

The total quantity of primary and secondary care prescriptions were extracted for the period paralleling the sampling months, at each WWTPs site. Prescription data from general hospitals within the catchment areas were included. However, prescription data from dental hospitals, private hospitals, and other non-medical prescribers (such as nurses and pharmacists) were not incorporated, as these data are not publicly available.

An average daily prescribed mass was calculated for each PPPD ( $\mathrm{kg} / \mathrm{month}$ ) by accounting for the number of days in the month (days) and a mass conversion of $\mathrm{kg}$ to $\mathrm{mg}$. This value was normalised for the catchment using the population estimate (PE-WW), to give population normalised daily prescription (PNDP), Equ.6.

Equation 6: $\quad P N D P\left[m_{\text {day }}^{-1} 1000\right.$ inh $\left.^{-1}\right]=10^{6}(P P P D) /$ days $/ 1000\left(P E_{W W}\right)$

\subsection{Experimental uncertainties}

For each stage of the methodology, the experimental features and limitations were considered. These are discussed throughout Table 4.

Table 4 Experimental uncertainties

\begin{tabular}{|l|l|l|l|}
\hline Variable & Aspect & Considerations & Comments on confidence \\
\hline \multicolumn{2}{|l|}{ Antimicrobial metabolite quantitation } & High. Assumes homogenised wastewater. \\
\hline Sample & Representation & $\begin{array}{l}\text { Samples were wastewater influent: an } \\
\text { amalgamation of waste from the contributing } \\
\text { community. Autosamplers were used: 24hr } \\
\text { composite samples (flow proportional collection, } \\
\text { every 15 min). }\end{array}$ & \\
\hline
\end{tabular}




\begin{tabular}{|c|c|c|c|}
\hline & Integrity & $\begin{array}{l}\text { Analyte stability was tested under two } \\
\text { temperature conditions to assess potential } \\
\text { degradation throughout the sampling period. }\end{array}$ & $\begin{array}{l}\text { Analyte dependent. Testing did not include analyte } \\
\text { stability prior to auto-sampling. Distance/duration, } \\
\text { temperature, and infrastructure might impact } \\
\text { analyte degradation and biotransformation to } \\
\text { varying degrees between catchments. }\end{array}$ \\
\hline \multirow{4}{*}{ Analysis } & Method & $\begin{array}{l}\text { The analytical method has been validated for the } \\
\text { quantitation of antimicrobials in wastewater } \\
\text { influent [26]. }\end{array}$ & $\begin{array}{l}\text { High, but analyte dependent. Limits of detection } \\
\text { ranged from } 0.002 \text { to } 3.6 \mu \mathrm{g} / \mathrm{L} \text {. Inter- and intra-day } \\
\text { assessments of accuracy ranged } 86.2-112.7 \% \text { and } \\
\text { precision of } 3.1-19.9 \% \text {. Analyte recovery ranged } \\
\text { from } 29.6-152.2 \% \text {. }\end{array}$ \\
\hline & Consumption & $\begin{array}{l}\text { Human consumption was distinguished from } \\
\text { disposal by concurrent quantitation of drug } \\
\text { metabolites. }\end{array}$ & $\begin{array}{l}\text { High. The selected antimicrobials are commonly } \\
\text { prescribed, meaning an acute increase of mass } \\
\text { load or change of metabolite ratio can be more } \\
\text { easily attributed to events of drug disposal. }\end{array}$ \\
\hline & \multirow{2}{*}{ Calculations } & $\begin{array}{l}\text { Flow rates were obtained from the corresponding } \\
\text { WWTP. These were averaged for a volume/day, } \\
\text { to calculate g/day. }\end{array}$ & High. \\
\hline & & $\begin{array}{l}\text { Population estimates were obtained from a water } \\
\text { utility. This was used to calculate } \\
\mathrm{mg} / 1000 \text { inh/day. }\end{array}$ & $\begin{array}{l}\text { High, but single annual value used. Does not } \\
\text { account for work/student/social commuting. }\end{array}$ \\
\hline \multicolumn{4}{|c|}{ Catchment prescription records } \\
\hline \multirow{7}{*}{ Meta data } & Source & Obtained from NHS & High. \\
\hline & & $\begin{array}{l}\text { Records document the date of prescription. This } \\
\text { may not represent the start date of antimicrobial } \\
\text { consumption. The full course (in mass) will also be } \\
\text { attributed to one date. }\end{array}$ & $\begin{array}{l}\text { Moderate. Conscious of possible misalignment } \\
\text { between prescription (pre-emptive) data and WBE } \\
\text { (measured) data. }\end{array}$ \\
\hline & Records & $\begin{array}{l}\text { Antimicrobials are available in different } \\
\text { presentation forms, i.e., active component with } \\
\text { varying doses, quantities, and medicinal form. }\end{array}$ & $\begin{array}{l}\text { Moderate. Total prescription quantity for each } \\
\text { active component in their presentation form were } \\
\text { calculated using PrAna, an R package. Briefly, Each } \\
\text { variation of the presentation is uniquely labelled } \\
\text { with a BNF/SNOMED code. These factors were } \\
\text { accounted for in the total mass calculation. Drug } \\
\text { production regulations ensure quality control, in } \\
\text { terms of acceptable quantities of active } \\
\text { component and impurities. }\end{array}$ \\
\hline & Catchment map & $\begin{array}{l}\text { Catchment map was provided by WWTP, } \\
\text { generated based on the sewage network. }\end{array}$ & Low. \\
\hline & $\begin{array}{l}\text { Selection of GP } \\
\text { surgeries }\end{array}$ & $\begin{array}{l}\text { GP Surgeries were selected based on the } \\
\text { Catchment map and GP surgery's locations using } \\
\text { PrAna, R package. }\end{array}$ & $\begin{array}{l}\text { Moderate. It is evident from NHS digital dataset } \\
\text { that drugs prescribed at a GP may be dispensed at } \\
\text { a pharmacy located outside the same WWTP }\end{array}$ \\
\hline & Interpretation & $\begin{array}{l}\text { Meta data was interpreted using PrAna, an } R \\
\text { package developed using freely available statistics } \\
\text { software package R. }\end{array}$ & : \\
\hline & Representation & $\begin{array}{l}\text { The selected antimicrobials are only available via } \\
\text { prescription. Both primary and secondary care } \\
\text { prescription data is included. }\end{array}$ & Moderate. \\
\hline \multirow{4}{*}{ Population } & $\begin{array}{l}\text { PE-WW } \\
\text { Estimation }\end{array}$ & $\begin{array}{l}\text { Calculations based on water utility estimates. } \\
\text { Briefly, resident population estimate was } \\
\text { calculated by multiplying number of properties by } \\
\text { occupancy rate, adjusted for care homes, } \\
\text { residential schools etc. The occupancy rate is set } \\
\text { at district level. }\end{array}$ & $\begin{array}{l}\text { High. This is based on the properties and cannot } \\
\text { be easily updated to accommodate variations } \\
\text { caused by births, deaths, and migration during the } \\
\text { sampling period. }\end{array}$ \\
\hline & $\begin{array}{l}\text { PE-NHS } \\
\text { Estimation }\end{array}$ & $\begin{array}{l}\text { Population estimates were calculated by the } \\
\text { number of registered people in the GP surgeries } \\
\text { located inside the WWTP catchment zone. The } \\
\text { registered population information for each GP } \\
\text { was obtained from NHS Digital. }\end{array}$ & $\begin{array}{l}\text { Moderate. It is possible that people registered in a } \\
\text { GP but live/work in a different postcode location. } \\
\text { It is monthly updated data, but still does not } \\
\text { consider fluctuations during sampling (e.g., } \\
\text { commuting patterns and holidays) }\end{array}$ \\
\hline & \multirow[t]{2}{*}{ Behaviour } & $\begin{array}{l}\text { Antibiotic courses should be taken in full. } \\
\text { However, it is known that individuals may stop } \\
\text { when their symptoms subside. }\end{array}$ & Moderate. \\
\hline & & $\begin{array}{l}\text { Prescriptions may be disposed of down-the-drain, } \\
\text { or not dispensed }\end{array}$ & Moderate \\
\hline
\end{tabular}




\begin{tabular}{|c|c|c|c|}
\hline \multirow{2}{*}{$\begin{array}{l}\text { Literature } \\
\text { review }\end{array}$} & Protocol & $\begin{array}{l}\text { A systematic review was designed to identify } \\
\text { relevant excretion research. }\end{array}$ & $\begin{array}{l}\text { High. The protocol was not fully comprehensive, } \\
\text { and so additional papers were included that were } \\
\text { identified from references and existing reviews. }\end{array}$ \\
\hline & Methodology & $\begin{array}{l}\text { Study design was varied throughout the } \\
\text { literature, including subject inclusion criteria; } \\
\text { dose regimen; administration type; sample } \\
\text { collection; and analytical methods used (method } \\
\text { performance). }\end{array}$ & $\begin{array}{l}\text { High. Experimental variables were documented, } \\
\text { and statistical analyses were performed to assess } \\
\text { the significance of each condition on metabolite } \\
\text { excretion. }\end{array}$ \\
\hline \multirow{2}{*}{ Calculation } & $\begin{array}{l}\text { Weighted } \\
\text { average }\end{array}$ & $\begin{array}{l}\text { Average analyte excretion was weighted by the } \\
\text { number of observations, per study. Studies } \\
\text { providing semi-quantitative/ insufficient data } \\
\text { were assigned a weighting factor of } 1 \text {. }\end{array}$ & $\begin{array}{l}\text { High. Weighting reduces the effects of outlying } \\
\text { data due to unusual study conditions, such as } \\
\text { concurrent medications. }\end{array}$ \\
\hline & Intake & $\begin{array}{l}\text { Correction factors, per analyte, were calculated. } \\
\text { Assessment of experimental conditions was used } \\
\text { to evaluate the significance of different variables. }\end{array}$ & $\begin{array}{l}\text { Moderate. Median result used. Assumes } \\
\text { representative population characteristics - i.e., } \\
\text { age, ailments, lifestyle, treatment regimens etc.; as } \\
\text { well as any bias derived from the review. }\end{array}$ \\
\hline \multicolumn{4}{|c|}{ Prescription back-calculation to community usage } \\
\hline $\begin{array}{l}\text { Application } \\
\text { in UK }\end{array}$ & Catchments & $\begin{array}{l}\text { Estimating prescription usage via WBE in UK } \\
\text { catchment areas can be validated against the } \\
\text { prescription database tool. }\end{array}$ & See manuscript Results \& Discussion section. \\
\hline
\end{tabular}

\subsection{Analyte stability study}

The stability of analytes in influent wastewater was assessed over $24 \mathrm{~h}$, as discussed in a previous publication [29]. A homogenised influent wastewater sample was split in physical replicate and stored at two temperature conditions: ambient room temperature $\left(18-21^{\circ} \mathrm{C}\right)$ and refrigerated temperature $\left(8-14{ }^{\circ} \mathrm{C}\right)$, SI fig.6. Aliquots were taken at six time points $(\mathrm{t}=0,3,6,16,20$, and $24 \mathrm{~h})$, spiked with internal standards $\left(25 \mu \mathrm{L}\right.$ of $\left.1 \mu \mathrm{g} \mathrm{mL} \mathrm{m}^{-1}\right)$, and processed via solid phase extraction. UPLC-MS/MS analysis was conducted as described in section 2.3.

Analyte stability was determined by relative concentration change during the $24 \mathrm{~h}$ time-period. The average of triplicate samples was taken for each time point, error bars representing the standard deviation. Analyte degradation was assessed in reference to drug class, as well as the impact of sample temperature.

\section{Results \& Discussion}

3.1. Drug excretion correction factors (CFs)

In order to calculate analyte-specific excretion factors, metabolite excretion data from published research were collated. This was achieved through a literature searching protocol, consisting of: (1) systematically obtaining a pharmacokinetic literature pool; (2) including/excluding papers based on experimental design via title and abstract; (3) including/excluding based on data in the main text; (4) calculating analyte weighted medians and correction factors.

\subsubsection{Literature review}

Adopting a systematic approach produced a comprehensive dataset from a vast number of journals, throughout a broad period of time (1938-2021). Literature hits were sorted using pre-set exclusion criteria, and the exclusions were recorded as an evaluation of the protocol efficiency (Figure 1). The most common exclusion was the experimental design/study type, for example those evaluating drug/regime efficacy; isolate testing/susceptibility; method development; analyte degradation; etc. Other papers were excluded when observing the 'wrong' analyte. These false positives occurred when the target antimicrobial was mentioned as an adjunctive/replacement/alternative medication; or when human biomarkers (creatine/potassium/uric acid/etc.) were being monitored alongside the administration of the target drug. The terms 'urinary' and 'pharmacokinetic' also produced false positives ${ }^{1}$ but were retained in the protocol to maximise the number of relevant papers found. Literature reviews were generally excluded to avoid duplication of results. However, in cases where the reviews referenced inaccessible/non-English research, the results are included and both papers noted. Literature found outside of the systematic protocol (labelled 'unsystematic') were also included. The literature not identified using the protocol was often older research, without digitised abstracts or keywords listed in the databases.

\footnotetext{
${ }^{1}$ Urinary: Several of the antibiotics studied are used in the treatment of urinary tract infections. Pharmacokinetics: the study of absorption, bioavailability, and distribution - as well as metabolism and excretion.
} 
Possible limitations to the experimental design used in the various studies are important to consider:

1. Sample: Each drug is metabolised and excreted via different pathways and so has varying washout patterns. Results may not report complete drug elimination if the sample collection does not include both urine and faeces or is not for a long enough period after ingestion.

Urinary and faecal samples were used in calculations, however additional data for other excretions, such as bile and ileostomy effluents, are quoted in SI tab.6.

2. Regimen and administration: The drug regimen can also have significant impact on the excretion of metabolites. Many of studies involve the comparison of various treatment protocols. These include dose quantity and regularity; fasting/fed conditions; the addition of concurrent medication; and the drug form/method of administration. Topical applications were highlighted due to a small presence detectable in urinary and faecal samples.

All drug regimens and forms of administration were included. Most of these variables had no substantial effect on analyte excretion, however some concurrent medications were observed to reduce the quantity measured in urine \&/ faeces. For example, several studies of ciprofloxacin observed the significant impact of antacids and other medications containing metal ions, such as ferrous sulfate [30], calcium carbonate [31], aluminium or magnesium [32]. More than $80 \%$ of the papers identified for nitrofurantoin specified whether the drug regimen was administered under fasting or fed conditions, and seven involved studies that directly compared this variable. Individually, these latter studies observed a lower percentage excretion under fasting conditions of 0-22\%, relative to fed participants [33-39]. However, once all the collated data was considered, this significance of this variable became negligible $(38.7 \%$ weighted average for fed, versus $38.4 \%$ for fasting). The route and form of administration can influence the pharmacokinetics of a drug, e.g., oral tablets, oral suspensions, intravenous injection, topical cream, modified release, etc. The most common administration was oral tablets/capsules, followed by intravenous injection (i.v.).

3. Subjects: Collated data was focussed predominantly on human excretion, with the exception of a small number of papers measuring animal excretion rates. These were only included in the absence of equivalent human data, such as faecal excretion of trimethoprim [40]. Drug excretion can vary with health, age, gender, lifestyle, etc. Thus, all results reference the type of participant studied, i.e., healthy volunteers, patients (generic), and patients (renal failure).

The studies involved a broad mix of subjects. The most significant impact on analyte excretion was a patient's renal function; where for several of the targeted drugs, patients suffering from renal failure had significantly reduced excretion. Another factor that many papers observed, was the characterisation of 'fast' and 'slow' acetylators [41-43]. Sulfonamides, for instance, metabolise by acetylation. This variable was not monitored in the review. Due to the high volume of papers and participants studied, the final excretion values should be quite representative of the phenotypic diversity within the population.

4. Analytical methods: Numerous assays were utilised for each analyte. The accuracy and specificity of which varied significantly. Sample preparation/analysis could not always distinguish unchanged and metabolite forms, such as glucuronides. These distinctions are highlighted in SI tab.6.

The literature studied used many different quantitation techniques, such as UV or mass spectrometry (structure/mass, i.e., metabolite specific); versus radiolabelling or bioassays (structure/microbiological activity, i.e., often non-specific). Several drug metabolites have degrees of antibacterial activity. Therefore, the lower specificity in bioassays could be expected to lead to over-estimations of percentage excretion. This trend is seen within the literature review for clindamycin and ciprofloxacin. However, the antibiotic-activity contribution of metabolite forms is often negligible. For these drugs, one would expect reasonable concordance between HPLC and bioassay analyses. For example, ofloxacin in literature [Dagrosa 1986, Ichihara 1984], as well as our collated data for ciprofloxacin, ofloxacin, and trimethoprim (SI fig.4-5). Alternatively, for most, the excretion percentage was found to be lower via methods using bioassays rather than chemical assays. For doxycycline, a median excretion of $40.3 \%$ was calculated for chemical assays and $18.7 \%$ for bioassays. This was attributed, in part, to a several of the bioassay studies administering concurrent medication or observing patients with renal failure, lowering the resultant excretion value. By removing these studies, the medians became $41.3 \%$ and $26.6 \%$. The remaining difference was unexplained by the other variables observed. It is possibly due to differences in method sensitivity. The results obtained via all assay types were included in the calculations of drug excretion, except in tetracycline and doxycycline. For these two drugs, studies using bioassays presented significantly lower percentage excretion, and so were excluded from the median calculations. 


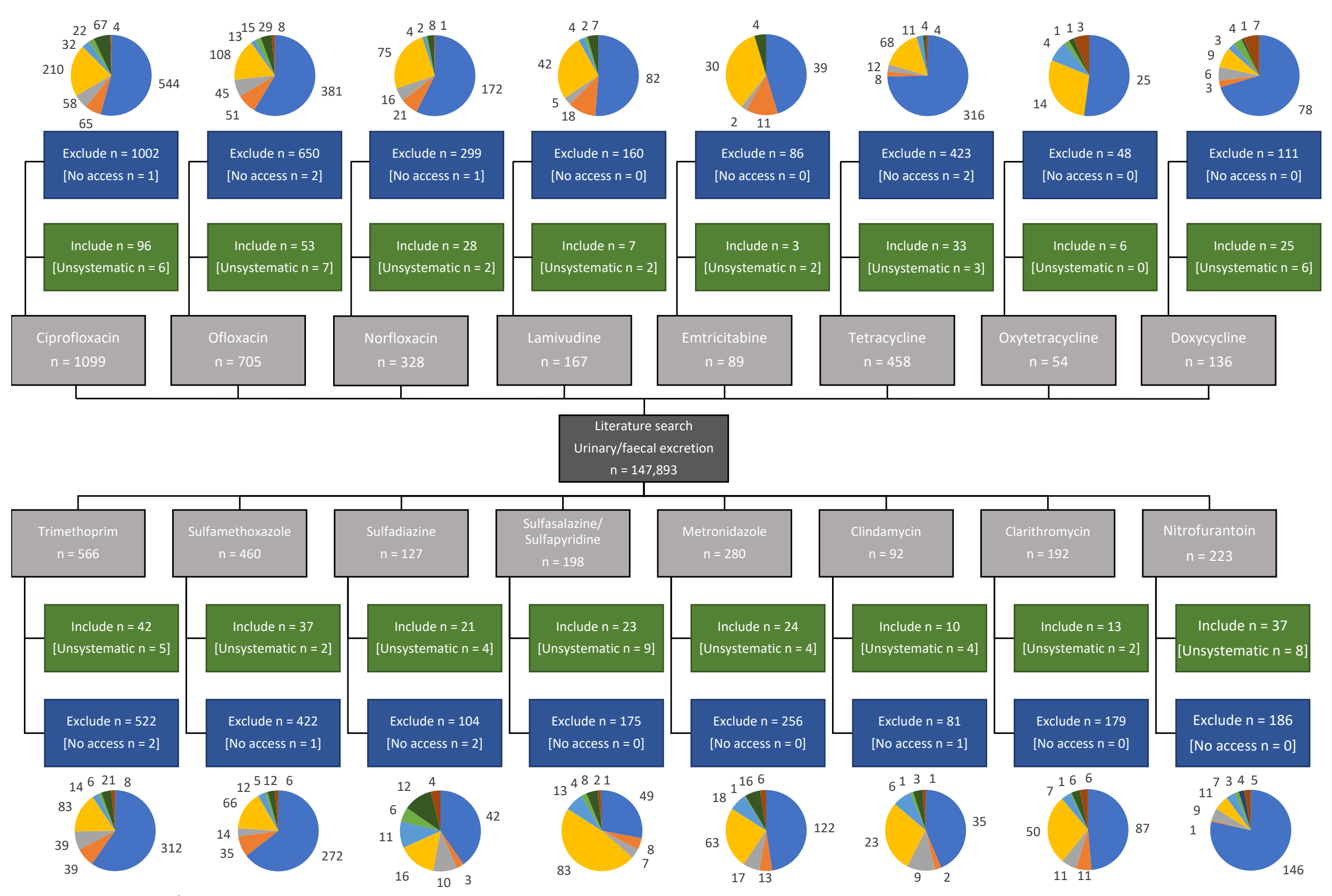

Figure 1 Literature search summary

Drug excretion literature pool and subsequent antimicrobial-specific results. Pie charts display the reasons for paper exclusion: study type (dark blue); matrix analysed (orange); review paper (light grey); analyte studied (yellow); animal study (light blue); replicate listing (light green); insufficient data (dark green); language (red); retracted (dark grey). A full break down of the search terms used is available in SI tab.5. 


\subsubsection{Data weighting}

Percentage excretions were collated per analyte and datapoints were assigned as either quantitative or semiquantitative. Semiquantitative labels were assigned for approximate/range values; values extracted from figures; and for studies with missing information, such as the number of subjects. The averaged percentage excretion across all studies was weighted by the number of observations made. For example, in the case of four participants, in a crossover study with two variables, the weighting factor would be 8 . However, if the datapoint was labelled as semiquantitative, the weighting factor was capped at 1 (Figure 2). The resultant weighted datasets are displayed, per metabolite, in Figure 3.

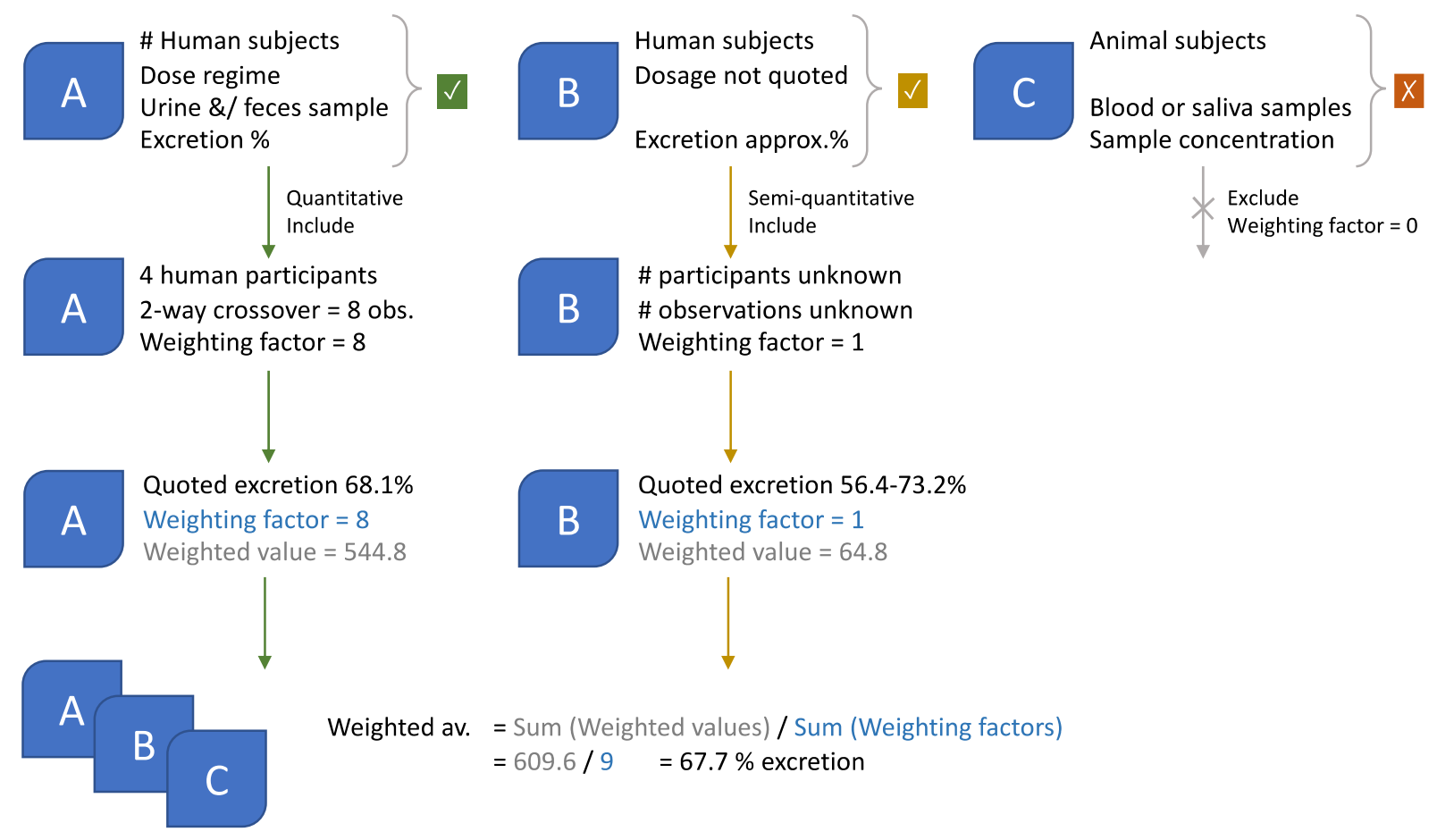

Figure 2 Literature weighting protocol, by study design and particularly the number of observations

\subsubsection{Correction factors}

Correction factors (CFs) were used to back-calculate the mass of each antimicrobial consumed in the community, via the analyte masses detected (PNDL, $\mathrm{mg} / 1000 \mathrm{inh} / \mathrm{day}$ ) as well as the rate and proportion of metabolite/s excreted. The values in Table 5 display the collated urinary/faecal excretion data and associated CFs, per metabolite. Metabolic and elimination pathways vary between different pharmaceuticals. Consequently, the proportion of a drug being excreted in urine or faeces will be analyte specific. The values quoted in the table are the weighed medians of urinary plus faecal excretion. Not all analytes are excreted via faeces, and there are fewer studies conducted overall that include faecal analyses. Therefore, data including faecal excretion are marked ( $\$)$, for clarity. A comprehensive breakdown of all literature results alongside selected experimental conditions is collated in SI tab.6.

Table 5 also demonstrates the relative significance of including/excluding glucuronides in the analyses. This is seen most significantly in hydroxy metronidazole, Figure 4. The significances of other experimental conditions were also evaluated. The most common routes of drug administration, oral and i.v., were typically comparable, except in the case of ciprofloxacin (CIP) and metabolite (deCIP). Here, i.v. administration recorded significantly higher urinary excretion (CIP 56.9\%, deCIP 3.1\%) than oral (CIP 33.0\%, deCIP 1.9\%), Figure 5. Other examples are outlined in section 3.1.2. 

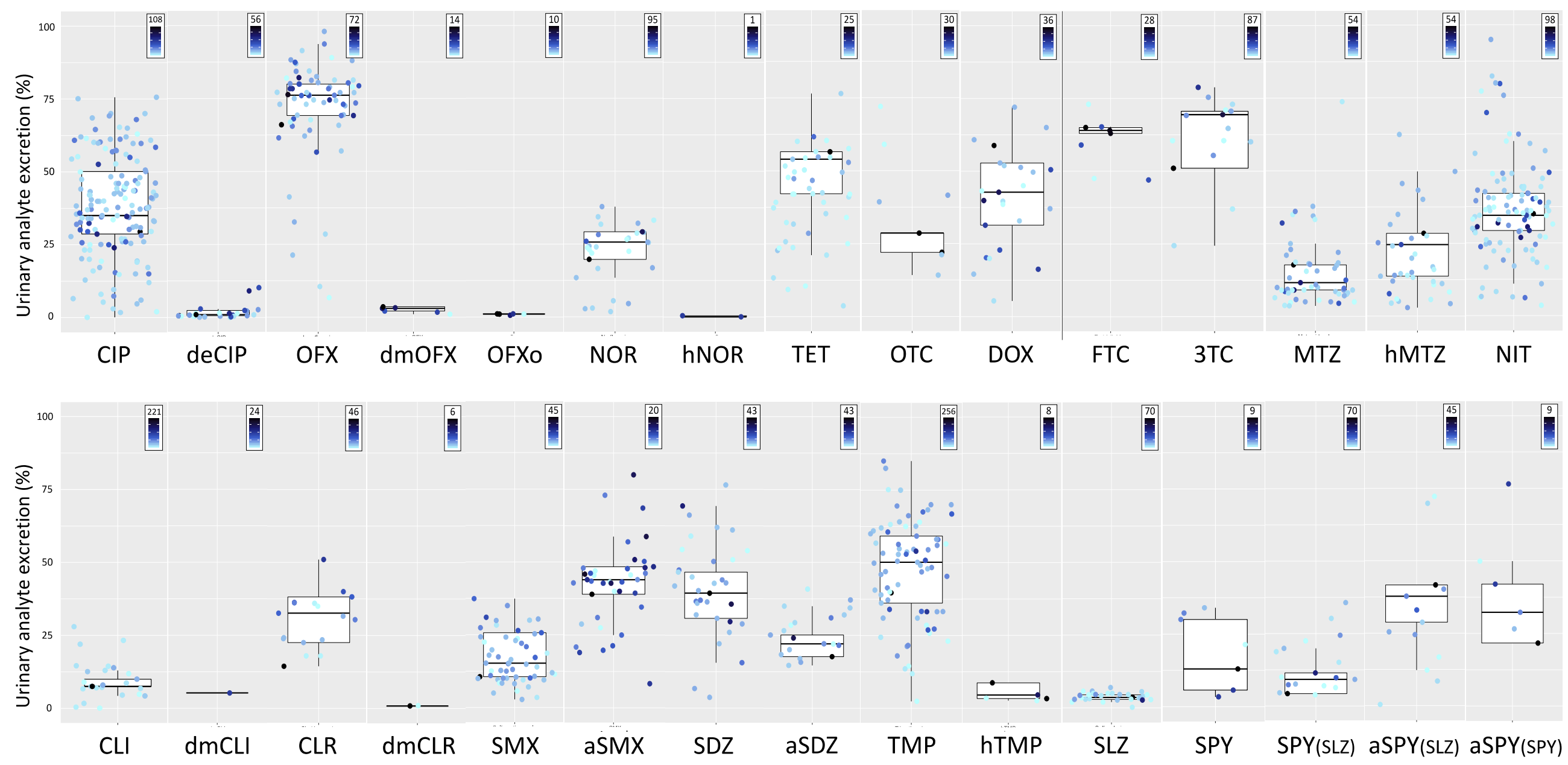

Figure 3 Weighted boxplots for percentage urinary excretion. The legend describes the range for the number of observations obtained by each study (which was used as the weighting variable). 
Table 5 Collated median human excretion rates and associated correction factors. Referenced literature available in SI tab. 6

\begin{tabular}{|c|c|c|c|c|c|}
\hline \multirow[b]{2}{*}{ Metabolite } & \multirow[b]{2}{*}{ Abbrev. } & \multirow{2}{*}{$\begin{array}{c}\text { Observations } \\
\mathrm{n}\end{array}$} & \multicolumn{2}{|c|}{ Observation-weighted urinary \&/ faecal excretion } & \multirow{2}{*}{$\frac{\text { Associated correction factor }}{\text { CF }}$} \\
\hline & & & Median excretion, \% & Ratio-excretion (P/M) & \\
\hline Sulfamethoxazole & SMX & 451 & 15.21 & & 6.57 \\
\hline Acetyl sulfamethoxazole & aSMX & 326 & 43.67 & 0.35 & 1.96 \\
\hline Trimethoprim & TMP & 641 & $63.09 \ddagger$ & & $1.58 \ddagger$ \\
\hline Hydroxy trimethoprim & hTMP & 24 & 3.83 & 16.49 & 24.77 \\
\hline Sulfadiazine & SDZ & 329 & 37.26 & & 2.68 \\
\hline Acetyl sulfadiazine & aSDZ & 184 & 22.05 & 1.69 & 3.88 \\
\hline Sulfasalazine ${ }^{\dagger}$ & SLZ & 262 & $15.99 \ddagger$ & & $6.25 \ddagger$ \\
\hline Sulfapyridine ${ }^{\dagger}$ & SPY [SLZ] & 244 & $13.37 \ddagger$ & 1.20 & $11.96 \ddagger$ \\
\hline Acetyl sulfapyridine ${ }^{\dagger}$ & aSPY [SLZ] & 120 & 37.75 & 0.42 & 3.62 \\
\hline Sulfapyridine ${ }^{\dagger}$ & SPY $¥$ & 28 & 21.40 & & 4.67 \\
\hline Acetyl sulfapyridine ${ }^{\dagger}$ & $\operatorname{aSPY}[S P Y] ¥$ & 25 & 29.43 & 0.73 & 2.91 \\
\hline Metronidazole & MTZ & 459 & $25.10 \ddagger$ & & $3.98 \ddagger$ \\
\hline Hydroxy metronidazole & hMTZ & 263 & 24.48 & 1.03 & 3.66 \\
\hline Clindamycin & $\mathrm{CLI}$ & 556 & $11.02 \ddagger$ & & $9.07 \ddagger$ \\
\hline Desmethyl clindamycin & $\mathrm{dmCLI}$ & 24 & 5.27 & 2.09 & 19.62 \\
\hline Clarithromycin & CLR & 198 & $34.28 \ddagger$ & & $2.92 \ddagger$ \\
\hline Desmethyl clarithromycin & $\mathrm{dmCLR}$ & 7 & $5.60 \ddagger$ & 6.12 & $18.20 \ddagger$ \\
\hline Ciprofloxacin & $\mathrm{CIP}$ & 2676 & $51.16 \ddagger$ & & $1.99 \ddagger$ \\
\hline Desethylene ciprofloxacin & deCIP & 415 & $1.41 \ddagger$ & 36.33 & $77.08 \ddagger$ \\
\hline Ofloxacin & OFX & 1364 & $80.00 \ddagger$ & & $1.25 \neq$ \\
\hline Desmethyl ofloxacin & dmOFX & 48 & $3.56 \ddagger$ & 22.47 & $29.23 \ddagger$ \\
\hline $\mathrm{N}$-oxide ofloxacin & oOFX & 34 & 1.00 & 3.56 & 95.76 \\
\hline Norfloxacin & NOR & 505 & $41.60 \ddagger$ & & $2.33 \neq$ \\
\hline Hydroxy norfloxacin & $\mathrm{hNOR}$ & 2 & 0.05 & 832.05 & 1904.57 \\
\hline Lamivudine & $3 \mathrm{TC}$ & 312 & 69.15 & & 1.45 \\
\hline Emtricitabine & FTC & 120 & $77.30 \ddagger$ & & $1.29 \ddagger$ \\
\hline Oxytetracycline & OTC & 81 & 22.55 & & 4.43 \\
\hline Tetracycline & TET & 904 & $64.52 \ddagger *$ & & $1.55 \neq$ \\
\hline Doxycycline & DOX & 233 & $81.65 \neq *$ & & $1.22 \ddagger$ \\
\hline Nitrofurantoin & NIT & 1449 & 34.49 & & 2.90 \\
\hline $\begin{array}{l}\text { 1-(2-nitrobenzylidenamino)-2,4- } \\
\text { imidazolidinedione }\end{array}$ & NPAHD & NA & NA & NA & \\
\hline
\end{tabular}

Excluding papers that only quote combined 'total' metabolite excretion ( + ); SPY no longer prescribed to humans as parent drug, therefore CFs not utilised in this manuscript ( $¥$ ); including faeces data

$(\ddagger)$; excluding bioassay data $\left({ }^{*}\right)$; parent drug $([$ Abbrev. $])$; total number of observations $(\mathrm{n})$; parent drug/metabolite $(\mathrm{P} / \mathrm{M})$. 
Table 6 Collated median human excretion rates and associated correction factors, separating to inclusion/exclusion of glucuronides in assays. Referenced literature available in SI tab.6

\begin{tabular}{|c|c|c|c|c|c|c|}
\hline \multirow[b]{2}{*}{ Metabolite } & \multirow[b]{2}{*}{ Abbrev. } & \multicolumn{3}{|c|}{ Observation-weighted urinary \& / faecal excretion, \% } & \multicolumn{2}{|c|}{ Associated correction factor (CF) } \\
\hline & & Inc Gluc. (n) & Exc Gluc. (n) & Sig. & Inc Gluc. & Exc Gluc. \\
\hline Sulfamethoxazole & SMX & $23.70(86)$ & $14.70(365)$ & $p<0.001$ & 4.22 & 6.80 \\
\hline Sulfapyridine ${ }^{\dagger}$ & SPY [SLZ] & $14.47(133) \ddagger$ & $8.37(111) \ddagger$ & $p=0.002$ & $11.04 \ddagger$ & $19.10 \ddagger$ \\
\hline Acetyl sulfapyridine ${ }^{\dagger}$ & aSPY [SLZ] & $39.47(106)$ & $8.46(14)$ & $p<0.001$ & 3.47 & 16.17 \\
\hline Sulfapyridine ${ }^{\dagger}$ & SPY $¥$ & $21.40(1)$ & $8.53(27)$ & $\mathrm{p}=0.616^{\wedge}$ & 4.67 & 11.72 \\
\hline Acetyl sulfapyridine ${ }^{\dagger}$ & $\operatorname{aSPY}[\mathrm{SPY}] ¥$ & $49.90(1)$ & $28.48(24)$ & $\mathrm{p}=0.619^{\wedge}$ & 1.71 & 3.01 \\
\hline Metronidazole & MTZ & $31.47(161) \neq$ & $23.35(298) \ddagger$ & $p<0.001$ & $3.18 \ddagger$ & $4.28 \mp$ \\
\hline Hydroxy metronidazole & hMTZ & $27.97(159)$ & $13.79(104)$ & $p<0.001$ & 3.20 & 6.49 \\
\hline
\end{tabular}

Excluding papers that only quote combined 'total' metabolite excretion ( + ); SPY no longer prescribed to humans as parent drug, therefore CFs not utilised in this manuscript ( $¥)$; including faeces data (¥); ANOVA Tukey stated no significant difference (^). Parent drug ([Abbrev.]); glucuronides (Gluc.); total number of observations $(\mathrm{n})$. 

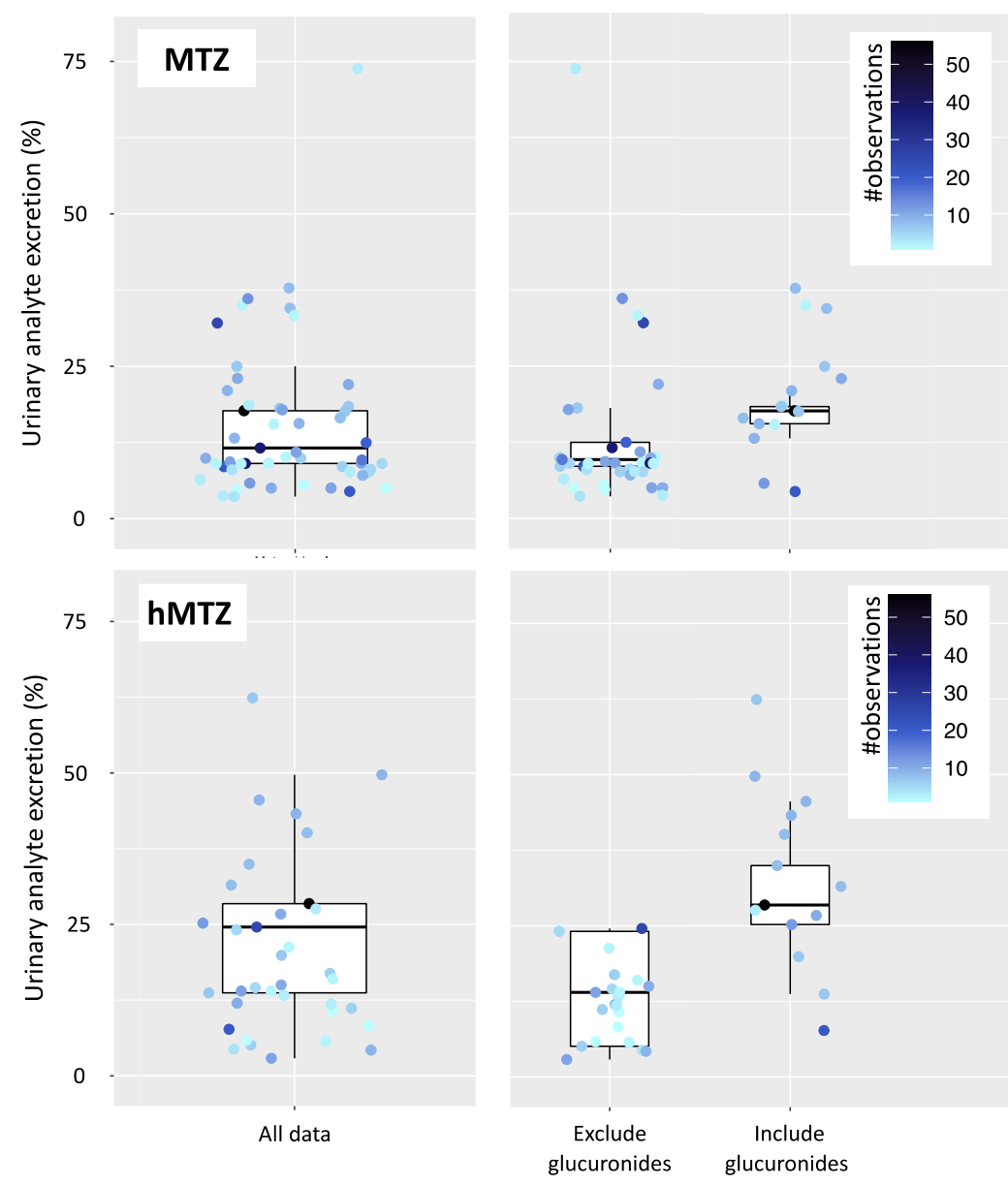

Figure 4 Example distribution of urinary excretion data for metronidazole for differing experimental conditions, weighted by number of observations
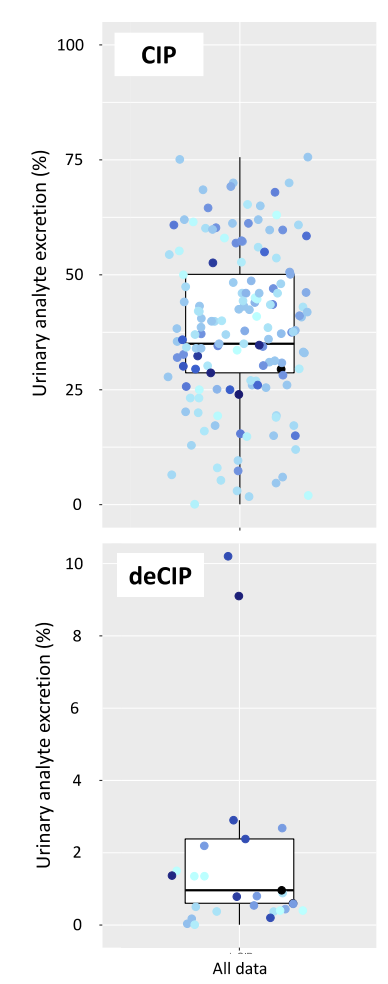
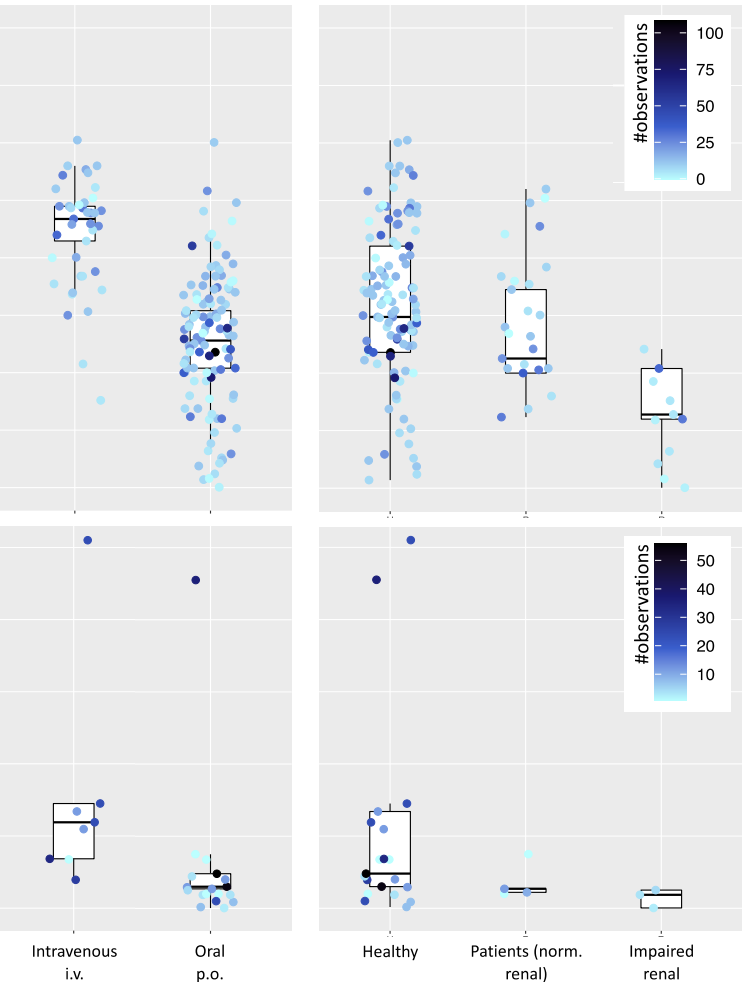

Figure 5 Example distribution of urinary excretion data for ciprofloxacin for differing experimental conditions, weighted by number of observations 


\subsubsection{Comparison of excretion rates used in this study and in published literature}

There are examples of published research that have used excretion data to calculate human intake as well as those that utilise prescription records alongside WBE data (Table 7). However, most of these studies only reference 15 excretion sources per drug and rarely quote the primary source or methodological conditions. Table 7 shows reasonable concordance between excretion values used between the different studies, however there are several broad ranges quoted.

\subsection{Analyte stability and method recovery}

The stability study displayed variation between the analytes as well as the two temperature conditions, Figure 6 . The sulfonamides were generally stable throughout the experiment, whereas their acetyl metabolites had decreased in concentration by approximately $10-30 \%$ after $24 \mathrm{~h}$, relative to $\mathrm{t}=0 \mathrm{~h}$. Trimethoprim, clarithromycin and the antiretrovirals were also largely stable throughout the $24 \mathrm{~h}$. Metronidazole exhibited a $20.7 \%$ reduction in the fridge condition, compared to $39.7 \%$ at room temperature in both parent and metabolite form. Tetracycline and oxytetracycline displayed an approximate 75 and 55\% decrease, respectively, independent of storage temperature. Doxycycline was poorly recovered from wastewater during method validation (30 $\pm 13 \%$, [26]). This may explain the high standard deviation and lack of degradation trend. Only 20-50\% of the starting sample concentration was recovered in clindamycin and its desmethyl metabolite. The largest decrease was observed between the first two time points, suggesting some of the initial loss may have been due to sorption to solids or the plastic container. This effect would not have been corrected for by ISTDs, as they were spiked per time point, just before solid-phase extraction. Although distinct losses were also noted in nitrofurantoin and NP-AHD (metabolite), the concentration decrease continued progressively throughout the study, suggesting degradation rather than adsorption. NP-AHD demonstrated significant temperature-dependent degradation $(78.0 \%$ versus $24.0 \%$ recovery). The quinolones did not demonstrate any class-specific trend: ciprofloxacin was recovered consistently throughout the study, whereas its metabolite, deCIP, was only recovered at $40 \%$ after 24 hours. Ofloxacin increased in concentration in both room and refrigerated conditions (511.4 and $231.7 \%$, respectively). Analyte carryover was negligible ( $<\mathrm{IDL})$ and internal standard acquisition was consistent. Therefore, the increasing mass may have been due to biotransformation - perhaps from the demethyl metabolite, which observed losses over the 24-hour period. Biotransformation could also explain the trend in norfloxacin. The hydroxy metabolite was largely stable throughout the study (85-120\% recovered); however, five other metabolites have been identified [44], not monitored in this study, that may have converted back into the parent form.

Matrix recoveries were conducted during the validation of the SPE-UPLC-MS/MS method [26], SI fig.8. The percentage recovery from WW is relevant for the estimation of daily drug intake (DI). This data was applied qualitatively to interpret the DI results. 
Table 7 Human drug intake research: referenced sources for urinary $(U)$, faecal $(F)$ and total $(T)$ excretion rates

\begin{tabular}{|c|c|c|}
\hline Metabolite & Analyte excretion \% [ref] & Source type (\#) \\
\hline \multirow[t]{4}{*}{$\mathrm{CIP}$} & $\underline{51.2}$ (U\&F) [this study] & $\begin{array}{l}\text { Primary sources (100), pharmaceutical databases (1), pharmacokinetic reviews } \\
\text { (1) }\end{array}$ \\
\hline & 26.4-61.4 (U\&F) [45] & Pharmaceutical databases (2), literature reviews (1), primary sources (1) \\
\hline & $55[46]$ & Pharmaceutical book (1) \\
\hline & $\begin{array}{l}25-62(U) ; 15-33(F) \\
1-12(\text { conj. U) [47] }\end{array}$ & Pharmaceutical databases ( $\leq 4)$, primary sources (NS) \\
\hline \multirow[t]{2}{*}{ deClP } & $\underline{1.4}$ (U\&F) [this study] & Primary sources (15), pharmaceutical databases (1), pharmacokinetic reviews (1) \\
\hline & $1 \%(U)[47]$ & Pharmaceutical databases ( $\leq 4)$, primary sources (NS) \\
\hline \multirow[t]{3}{*}{ CLR } & $\underline{34.3}$ (U\&F) [this study] & Primary sources (14), pharmacokinetic reviews (1) \\
\hline & $22-40$ (U\&F) [45] & Pharmaceutical databases (2), primary sources (1) \\
\hline & $25[46]$ & Pharmaceutical book (1) \\
\hline \multirow[t]{2}{*}{$\mathrm{CLI}$} & $\underline{11.0}$ (U\&F) [this study] & Primary sources (14), pharmacokinetic reviews (1) \\
\hline & $10-14$ (U\&F) [45] & Pharmaceutical databases (2) \\
\hline \multirow[t]{3}{*}{ DOX } & 81.7 (U\&F) [this study] & Primary sources (31) \\
\hline & $41[46]$ & Pharmaceutical databases (1) \\
\hline & $35(\mathrm{~T})[48]$ & Pharmaceutical databases (1) \\
\hline \multirow[t]{3}{*}{ MTZ } & $\underline{25.1}$ (U\&F) [this study] & Primary sources (28) \\
\hline & $13-27$ (U\&F) [45] & Pharmaceutical databases (2), literature reviews (1) \\
\hline & $25(\mathrm{~T})[48]$ & Pharmaceutical databases (1) \\
\hline \multirow[t]{2}{*}{ hMTZ } & $\underline{24.5}(U)$ [this study] & Primary sources (17) \\
\hline & $60-80(U)[45]$ & Primary sources (1) \\
\hline \multirow[t]{4}{*}{ NOR } & $\underline{41.6}$ (U\&F) [this study] & Primary sources (30) \\
\hline & $30[46]$ & Pharmaceutical book (1) \\
\hline & $63[47,49]$ & Literature reviews (1) \\
\hline & $\begin{array}{l}23-34(\mathrm{U}) ; 30-40(\mathrm{~F}) \\
0 \text { (conj. urine) }[47]\end{array}$ & Pharmaceutical databases ( $\leq 4)$, primary sources (NS) \\
\hline \multirow[t]{2}{*}{ OFX } & $\underline{80.0}$ (U\&F) [this study] & Primary sources (59), pharmacokinetic reviews (1) \\
\hline & $80[46]$ & Pharmaceutical databases (1) \\
\hline \multirow[t]{5}{*}{ SMX } & $\underline{15.2}(U)$ [this study] & Primary sources (38) \\
\hline & $15-30(U)[45]$ & Pharmaceutical databases (1), literature reviews (1) \\
\hline & $15-30[50]$ & Literature reviews (2) \\
\hline & 20 (U); 0 (F); 15 (Gluc) [47] & Pharmaceutical databases ( $\leq 4)$, primary sources (NS) \\
\hline & $30(\mathrm{~T})[48]$ & Pharmaceutical databases (1) \\
\hline \multirow[t]{3}{*}{ aSMX } & $\underline{43.7}(U)$ [this study] & Primary sources (28) \\
\hline & $50-70(U)[45]$ & Pharmaceutical databases (1) \\
\hline & $60-65(U)[47]$ & Pharmaceutical databases ( $\leq 4)$, primary sources (NS) \\
\hline \multirow[t]{2}{*}{ SLZ } & $\underline{16.0}$ (U\&F) [this study] & Primary sources (32) \\
\hline & $0(\mathrm{~T})[51]$ & Pharmaceutical databases (1) \\
\hline \multirow[t]{2}{*}{ TET } & $\underline{64.5}$ (U\&F) [this study] & Primary sources (36) \\
\hline & $58[46]$ & Pharmaceutical databases (1) \\
\hline \multirow[t]{4}{*}{ TMP } & $\underline{63.1}$ (U\&F) [this study] & Primary sources (46), pharmacokinetic reviews (2) \\
\hline & $40-52$ (U\&F) [45] & Pharmaceutical databases (2), primary sources (1) \\
\hline & $80[46]$ & Literature reviews (1) \\
\hline & $43(\mathrm{~T})[48,50]$ & Pharmaceutical databases (1) / literature reviews (1) \\
\hline
\end{tabular}

'Total' refers to Khan 2004, 'excreted biliary/faecal/renal unchanged'; not stated (NS); data from this study's literature review (bolded and underlined) 

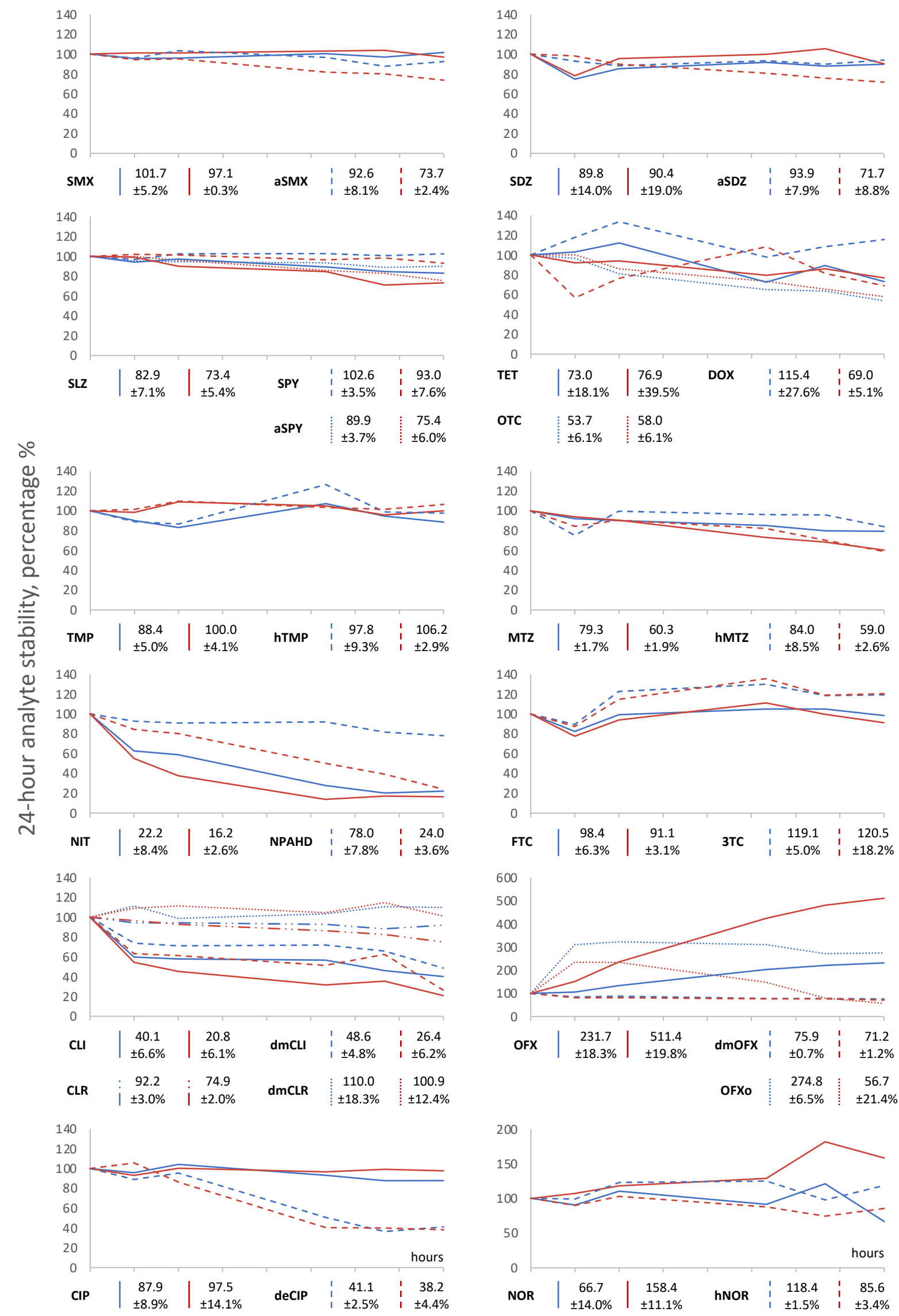

Figure 6 Analyte percentage stability (\%) versus time (0-24 hours)

Stability results are separated by parent drug and drug metabolites (line type), as well as displaying degradation at room temperature (red lines) versus refrigerated temperature (blue lines). Percentage of $\mathrm{t}=0$ analyte mass, present after 24 hours, is quoted under each plot, including the standard deviation from three physical duplicates. 


\subsection{Dataset evaluation}

Our methodology for obtaining and applying correction factors was evaluated using a high-resolution dataset of a catchment region in the SW of England against per-postcode pharma prescription records. Eight WW samples were randomly selected for winter months (Jan-Feb 2019) and eight for summer months (Jun-Jul), in order to observe temporal patterns. All data extracted for analysis were determined to be within analyte-specific quantitative range. Other validation criteria are described in SI spreadsheet1. Wastewater influent concentrations were processed via the equations outlined in section 2.5.2., using: daily WWTP influent flow; population estimates (Table 3); and CFs calculated from the median analyte excretion (Table 5). The resultant DI, per metabolite, were subsequently checked for concordance with prescription records associated with the catchment. The results of which are described in figures Figure 7Figure 8.

The interpretation of these results was achieved by considering each aspect of the process: Drug prescribed (PNDP); drug ingested, and metabolites excreted (CF); analyte stability in sewer system and composite sampler; analyte recovery from SPE-UPLC-MS/MS method; and data processed for the calculation of daily mass loads (DL), population-normalised loads (PNDL), and intake (DI).

The prescription data displayed in the figures are averaged population normalised daily prescription (PNDP, $\mathrm{mg} /$ day/1000inh), calculated from the total monthly mass of drug prescribed. However, drugs prescribed within the catchment may not be consumed consistently per day, throughout the month; may not be consumed at all (stored or disposed); may be consumed/excreted in surrounding catchment areas; or may be consumed across sequential months. Topical treatments will have different metabolic and excretion pathways, compared to enteral and parenteral. However, topical antibiotics are typically administered more frequently and at a lower dose, meaning any impact should be low and more consistent - relative to a disposal event, which would cause an acute effect on DI and the parent-metabolite ratio.

The corrections factors were calculated from a comprehensive pharmacokinetic literature review, weighted by the number of observations conducted by each study in order to achieve the most representative results. The weighted median was used in the calculation of DI. The mass of a drug ingested (DI) was back-calculated from the quantity of metabolite measured in WW and the proportion that the metabolite is excreted via urine/faeces. Consequently, each metabolite of a drug should theoretically calculate the same DI. However, this trend is not observed. This may be due to several reasons: relative metabolite stability and recovery; direct disposal of drugs; or metabolite biotransformation, such as glucuronide cleavage. The latter two examples would result in an over-estimate of the DI from the parent/unchanged form. This pattern is observed for SMX, SPY, MTZ, and CLI - all of which are known to form glucuronides. In these examples, the other metabolites being monitored often performed better for the calculation of DI, relative to PNDP.

Analyte stabilities and method recoveries were not included in the CF calculation. This was because the sewer hydraulic retention time and thermal conditions were not monitored; and analyte recovery was variable with sample matrix/composition. However, these variables were considered during the evaluation of the results. For instance, metabolites with lower stability and/or recovery may underestimate DI.

\subsubsection{Sulfonamides and trimethoprim}

Sulfamethoxazole and trimethoprim are often prescribed in combination, typically at a ratio of 5:1. However, the drug ratio in WW is not detected at these proportions due to physical-chemical and pharmacokinetic differences. A review by Thiebault et al. determined a median ratio of 1.93:1 [52]; and another study, using the same analytical methodology, calculated a median ratio of 3.8:1 [29]. The data used in this paper determined median PNDL-ratios of 1.4:1 and 2.1:1 in the eight winter and summer samples, respectively; but median DI-ratios of 5.8:1 and 8.8:1, respectively (SI fig.25). This demonstrated that the CFs used to calculate intake brought the drug proportions closer to the expected prescribed quantities. On the other hand, the corresponding prescription records suggested a much lower drug ratio (0.45:1). Figure 6 indicates that both sulfamethoxazole and trimethoprim are stable in wastewater. There are therefore potential other sources of deviation in drug ratio that require further investigation.

For sulfonamides, the acetyl metabolite is excreted as a large proportion of the drug (22-44\%); and for SMX and SLZ/SPY, these metabolites were determined to have a higher prevalence than the parent drug in urine/faeces. However, in WW the acetyl metabolite is typically detected at lower concentrations than the parent. This may be, in part, due to the lower relative stability observed in acetyl metabolites, Figure 6; but may also be due to glucuronide cleavage amplifying the parent form. Thus, the acetyl metabolites were typically better at estimating DI, when compared to PNDP (Figure 7).

The DI for sulfasalazine was more complex due to having three major metabolites: SLZ (unchanged form), SPY, and aSPY. Sulfapyridine is no longer prescribed to humans as a parent drug, and so only the CFs from SLZ-parent were applied. SLZ had the lowest recovery and stability of the three metabolites (possibly due to biotransformation) and consequently lowest DI; DIs from SPY were the highest (possibly due to glucuronidecleavage, and biotransformation from SLZ); and aSPY performed similarly to SLZ. Overall, SPY was determined to be the best predictor of SLZ DI, using a CF of 3.62. 
SDZ had a recovery from WW of $66.1 \%$, as the chromatography suffered from high matrix interference. This led to large inter/intra sample variance. The metabolite (aSDZ) was determined to be the best predictor of DI, using a $\mathrm{CF}$ of 3.88 .

TMP is predominantly excreted in the unchanged parent form $(63.1 \%)$, and only $3.8 \%$ is detected in urine for the metabolite, hTMP. This trend is observed in the WW data, as the metabolite is only detected sporadically and at low concentrations. The unchanged form, TMP, performed well in estimating DI (CF 1.58), when compared to PNDP.

\subsubsection{Metronidazole}

MTZ and hMTZ metabolites are excreted in near-equal proportions (25.1 and 24.5\%, respectively) and demonstrated equivalent thermal stabilities. Both analytes occur in a glucuronide form, but the proportion as glucuronide is most significant for hMTZ (Table 5). Glucuronide cleavage could over-estimate DI, whereas the low recovery of hMTZ (29.6\%) could under-estimate. Overall, when comparing to PNDP, hMTZ was considered a better predictor of DI, using a CF of 3.66 .

\subsubsection{Nitrofurantoin}

NPAHD was not monitored in any pharmacokinetic excretion studies, determined by the systematic protocol; therefore, no CF could be calculated. Due to the low stability of NIT (approx. 19\% after 24 hours), no mass was detected in the selected samples. Consequently, it was not possible to calculate DI, in this study.

\subsubsection{Lincomycins and macrolides}

In both CLI and CLR, the unchanged form was excreted at a higher proportion than the desmethyl metabolites (P/M ratio-excretion: CLI 2.1 and CLR 6.1, Table 5). The method recovery for dmCLI was lower than CLI, and so the ratio-WW was higher than ratio-excretion; the stability of dmCLR was greater than CLR, and so the ratioWW was lower than ratio-excretion (Figure 8).

Although none of the human studies in the literature review monitored CLI glucuronide conjugates, some animal studies have observed this as a metabolic route [53]. Direct disposal and/or glucuronide-cleavage could explain the overestimation of DI from the unchanged CLI form. CLR, on the other hand, performed well as estimating the DI.

Overall, dmCLI was the preferred metabolite for predicting clindamycin DI (CF 19.62) and unchanged CLR was preferred for CLR (CF 2.92) in this limited dataset. However, dmCLR should be still considered as a viable option in further studies. An overestimation of dmCLR was observed in a sample set used in this study with overestimation of DIs observed. There might be several reasons for it including inaccurate CF factors and PNDPs.

\subsubsection{Quinolones}

The quinolones are largely excreted in the unchanged form (CIP 51.2\%, NOR 41.6\%, and OFX 80.0\%), with a $<5 \%$ excretion of other metabolites. Consequently, the secondary metabolites were detected sporadically and at low concentrations. In the 16 samples selected for this evaluation, there was no detection of deCIP, OFXo, or hNOR; and only partial detection of dmOFX, relative to OFX. All quinolone analytes had method recoveries $>90 \%$ (except OFX, 73.2\%); however, stability results were erratic, Figure 6. DI calculated using the unchanged forms were comparable to PNDP, and therefore favoured (Figure 8).

Norfloxacin was not prescribed within the catchment during the four corresponding months. NOR parent drug was detected in WW, but sporadically and with high standard deviations between replicate samples. These data may be false positives, as all quoted results were detected at $\mathrm{S} / \mathrm{N}$ ratio of $<10$ (SI spr.1). No hNOR was detected in this case study.

\subsubsection{Antiretrovirals}

The majority of FTC and 3TC are excreted unchanged in urine and faeces (77.3 and 69.2\%, respectively). Metabolites were not monitored in this study, and so the two drugs were compared against each other. Analyte stability was high for both drugs, but the recoveries for FTC had a large inter-sample variance $(95.5 \pm 32.2 \%)$ and 3TC was only recovered at $31.5 \%$.

Both ARVs were detected at relatively high levels (up to $320 \mathrm{mg} / \mathrm{day} / 1000 \mathrm{inh}$ ). However, no prescriptions were stated in the catchment region. The drug levels detected in WW may have been prescribed outside of the observed catchment, or there may be other unaccounted for sources of ARVs in this study. Further work will be undertaken to understand ARV sources in the catchment.

\subsubsection{Cyclines}

The three cyclines monitored, (TET, OTC, and DOX), have varied excretion as the unchanged form $(64.5,22.6$, and $81.7 \%$, respectively). Metabolites were not monitored, and physical-chemical behaviour was as varied as the pharmacokinetic excretion. TET stability was approx. $75 \%$ after 24 hours and had an erratic, but high average 
recovery; OTC stability was approx. 56\% and poorly recovered from WW (47.3\%); and DOX had high sample variance during the evaluation of stability and very low method recovery $(29.6 \%)$. Due to this poor analyte recovery, no DOX was detected in the 16 selected samples and was therefore a poor biomarker for the estimation of DI.

The estimation of DI for TET (CF 1.55) and OTC (CF 4.43) was generally quite good, in reference to PNDP (Figure 8), despite the observed variance in physical-chemical behaviour.

\subsection{Temporal trends}

Eight samples were randomly selected from UK winter months, January and February, and eight from summer months, June and July. Figures 7 and 8 demonstrate the drugs that have seasonal trends - either due to consumption patterns or temperature dependant stability. Seasonal prescribing patterns should be observed in both PNDP and DI, whereas analyte degradation would only be observed in DI. An example of this is CLR, where DI is significantly lower during the summer months, compared to the winter. Whereas for CLI, DI and PNDP have inverse seasonal trends, which may be explained by higher degradation due to a relatively low thermal stability. Averaged summer and winter results are displayed in SI fig.14-15. However, due to the small number of samples observed and high standard deviations in several analytes, these trends are speculative and more extensive analyses are required. 
Population normalised mass loads (PNDLs)

SMX || $15.2 \% \quad$ aSMX $\| 43.7 \% \quad \frac{\text { SMX }}{\operatorname{aSMX}}$ ○

200

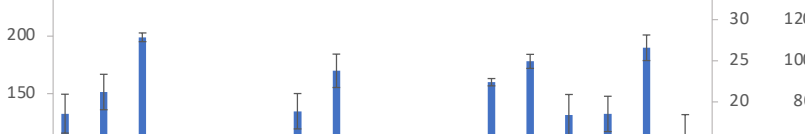

50 ○
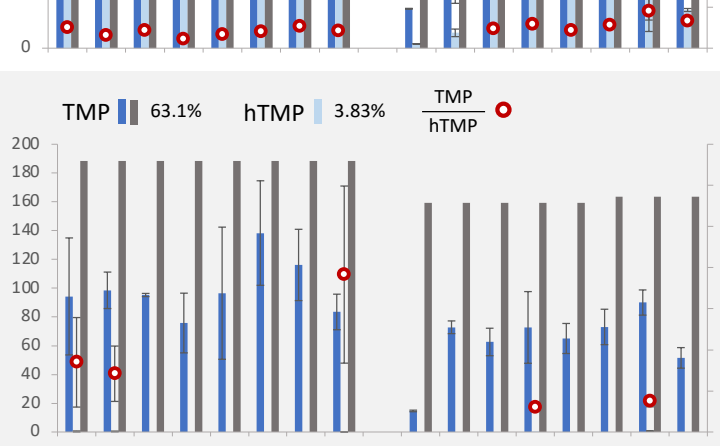

SLZ || $16.0 \%$ SPY | $13.4 \%$ aSPY $\| 37.8 \% \quad \frac{S L Z}{S P Y} \circ \frac{S L Z}{\text { aSPY }} \circ$

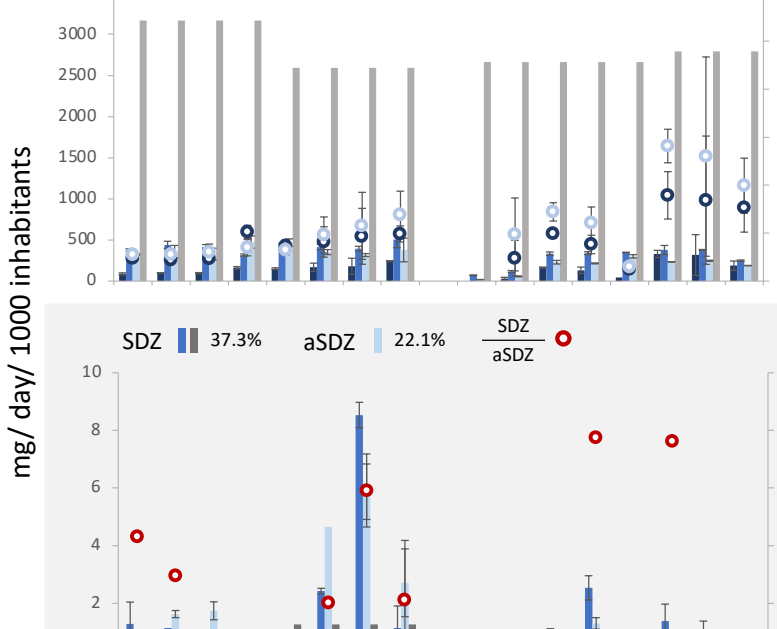

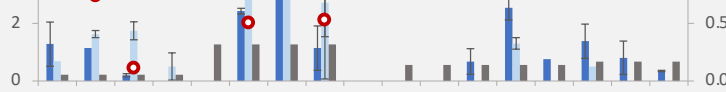
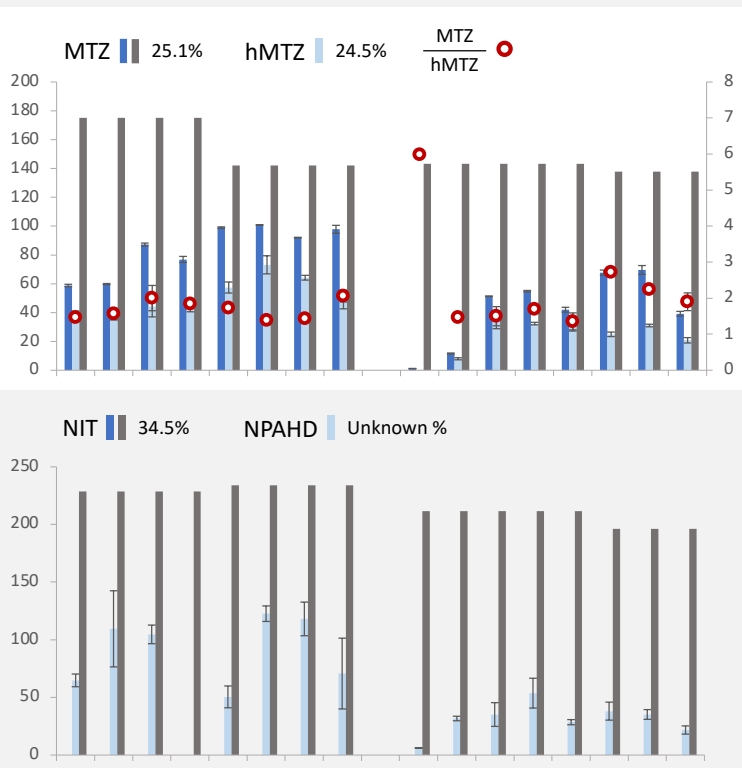

Winter

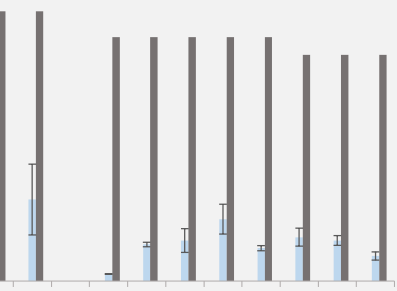

Summer

1200250
Daily intake (DI)

SMX || CF $6.57 \quad$ aSMX || CF $1.96 \quad \frac{\text { SMX }}{\text { aSMX }} \odot$

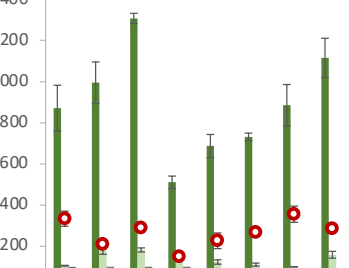

$\circ$

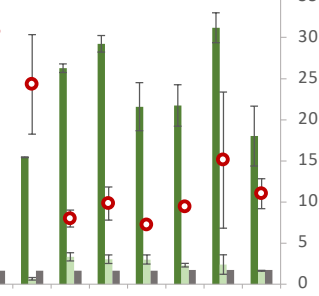

TMP || CF $1.58 \quad$ hTMP $\|$ CF $24.8 \frac{\text { TMP }}{\text { hTMP }}$ ○

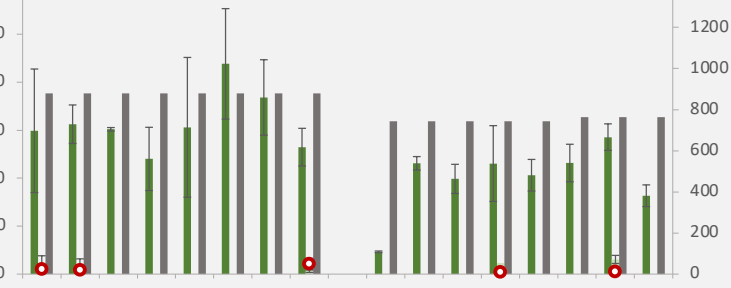

SLZ || CF 6.25 SPY | CF 12.0 aSPY | CF 3.62 $\frac{\text { SLZ }}{\text { SPY }} \odot \frac{\text { SLZ }}{\text { aSPY }} \circ_{-3.0}$
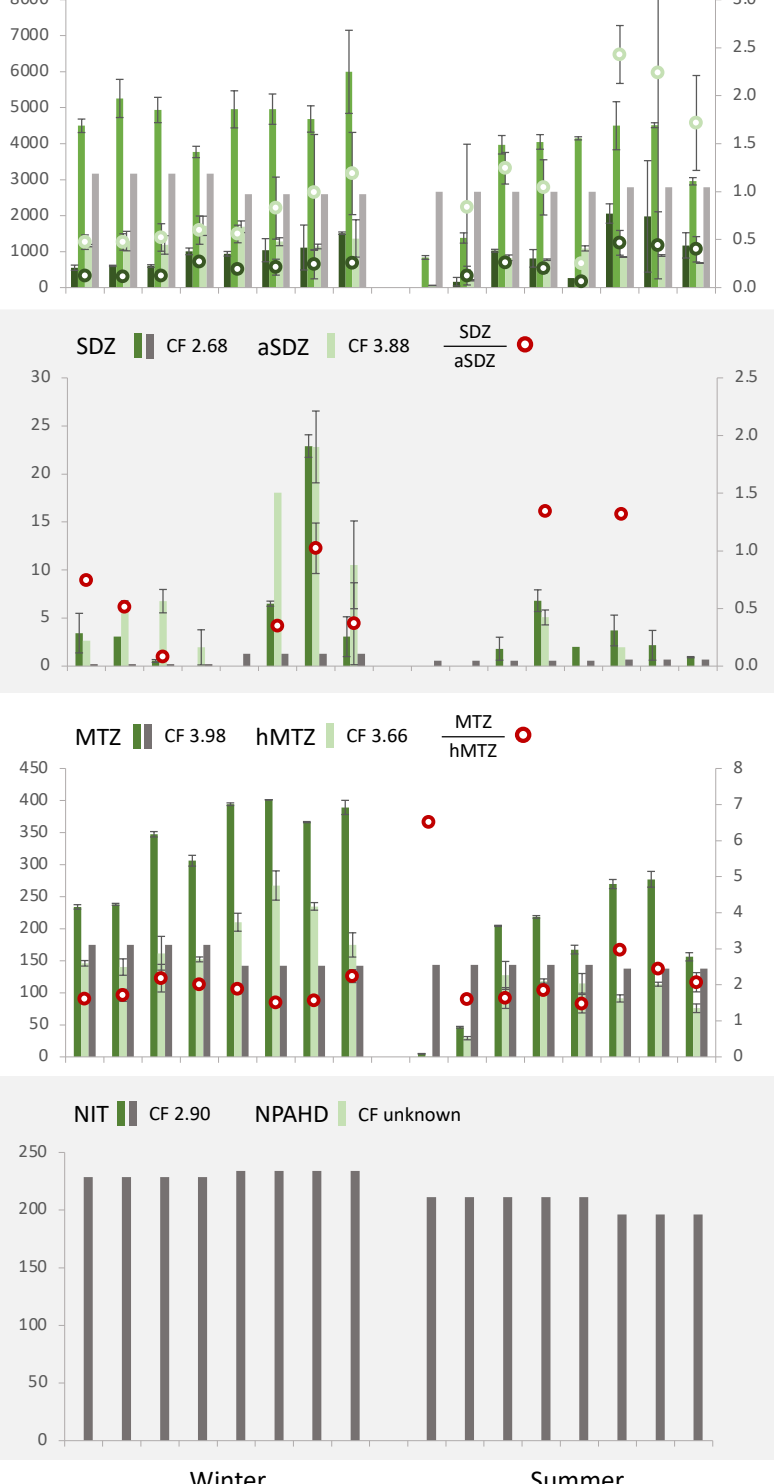

Figure 7 Daily drug mass results for sulfonamides, trimethoprim, metronidazole, and nitrofurantoin. PNDL (blues); DI (greens); PNDP (greys); analyte ratio-WW (circle markers). Enlarged figures available in SI fig..9-21. 
Population normalised mass loads (PNDLs)

CLI || $11.0 \% \quad$ dmCLI $\mid 5.27 \% \quad \frac{\mathrm{CLI}}{\mathrm{dmCLI}} \bigcirc$

$\mathrm{CLR}$ || $34.3 \% \quad \mathrm{dmCLR} \| 5.60 \% \quad \frac{\mathrm{CLR}}{\mathrm{dmCLR}} \mathrm{O}$

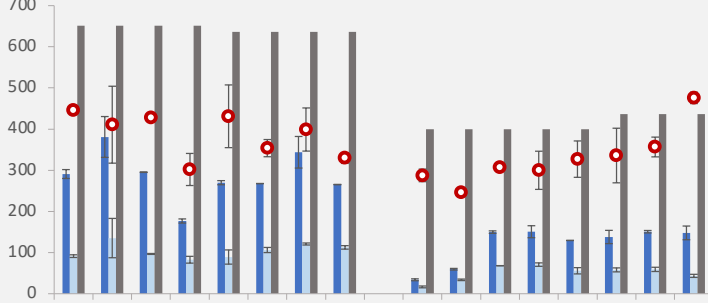

FTC || $77.3 \% \quad 3 T C|| 69.2 \% \quad \frac{\text { FTC }}{3 T C}$ -

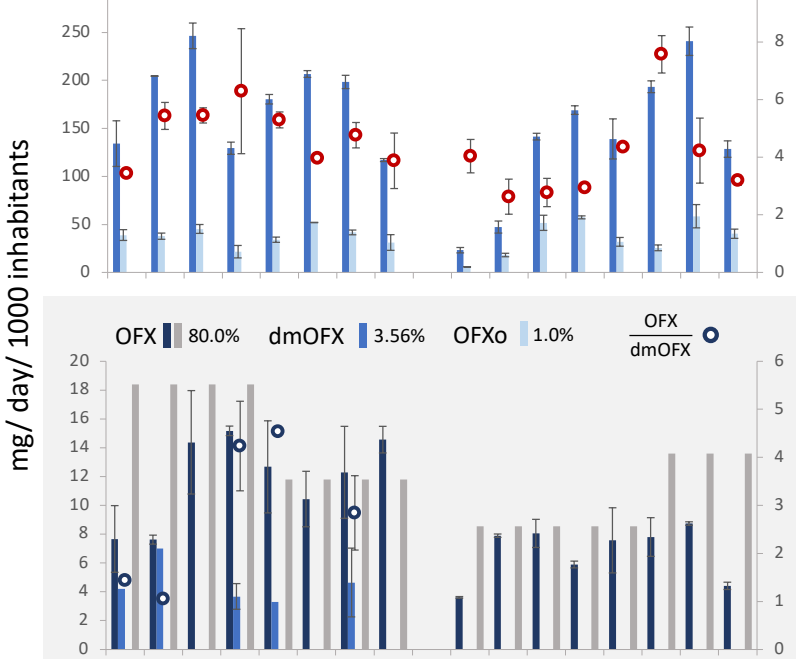

CIP || $51.2 \% \quad$ deCIP $\mid 1.41 \%$
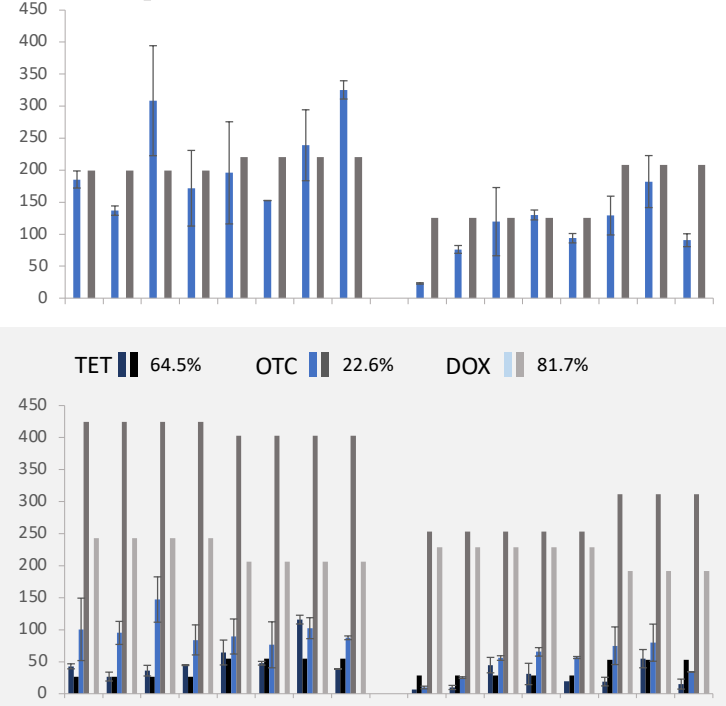

Winter

Summer

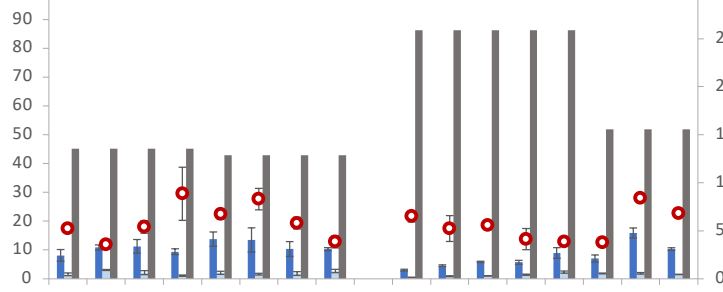

Daily intake (DI)

CLI I| CF $9.07 \quad \mathrm{dmCLI} \| \mathrm{CF} 19.6 \quad \frac{\mathrm{CLI}}{\mathrm{dmCLI}} \bullet$

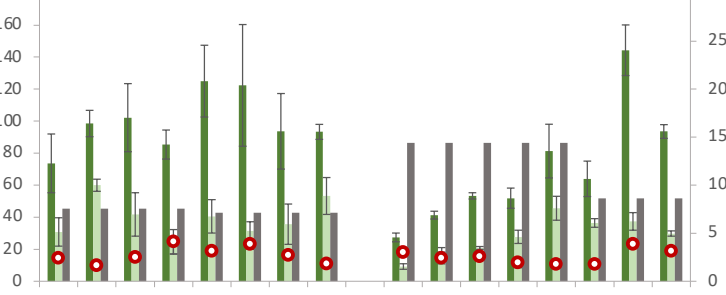

CLR \| CF $2.92 \quad \mathrm{dmCLR} \| \mathrm{CF} 18.2 \quad \frac{\mathrm{CLR}}{\mathrm{dmCLR}} \mathrm{0}$
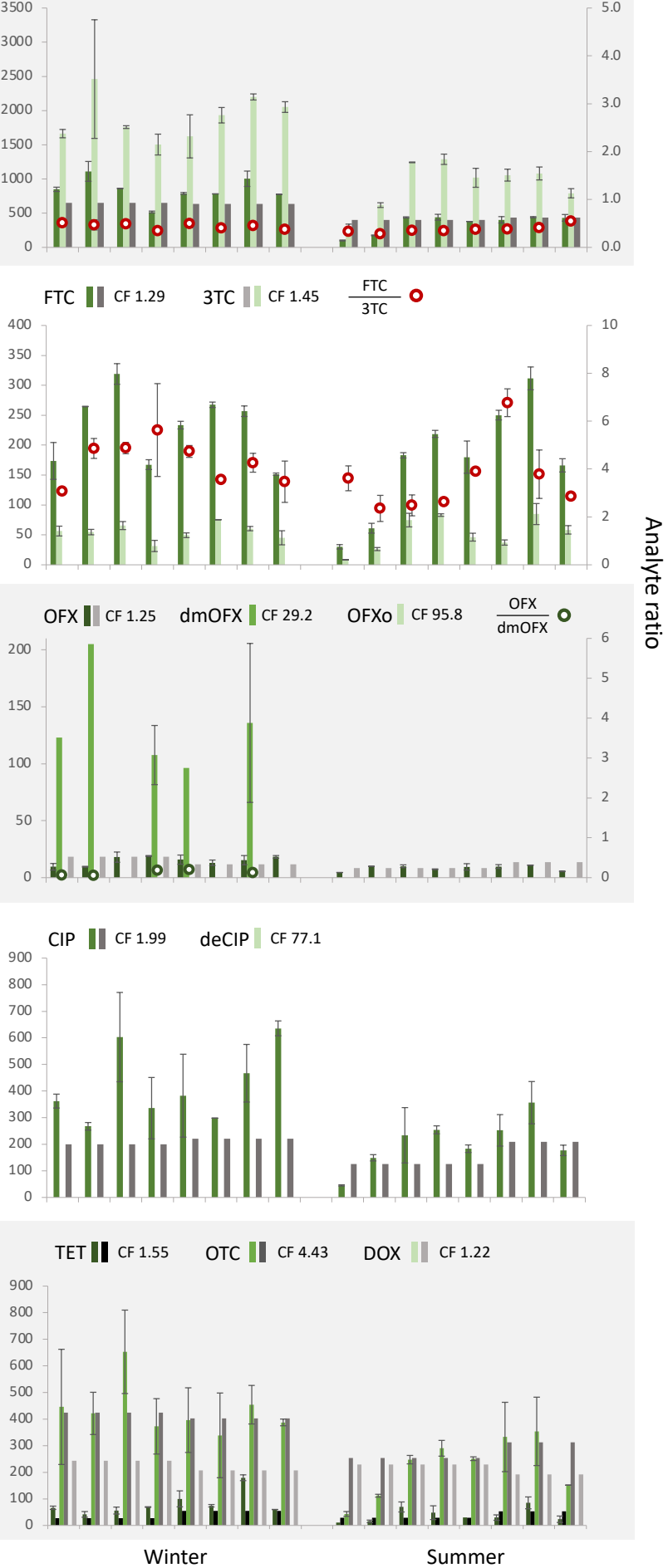

Figure 8 Daily drug mass results for macrolides, antiretrovirals, quinolones, and cyclines. PNDL (blues); DI (greens); PNDP (greys); analyte ratio-WW (circle markers). Enlarged figures available in SI fig.9-21. 


\section{Conclusions}

A comprehensive literature review of pharmacokinetic drug excretion studies was successful in establishing the proportion of metabolites expected in urine and faeces, after consumption. The median values were used to improve the estimation of daily community intake, calculated via wastewater-based epidemiology. Typically, WBE quotes population-normalised daily mass loads (PNDL); yet, unless a drug is fully eliminated, this will be an underestimate of total drug intake. This incidence was seen for many of the drugs (TMP, SLZ, MTZ, CLI, CLR, OFX, OTC, NIT) when comparing PNDL to catchment prescription records (PNDP). By correcting for metabolite excretion, the estimation of intake (DI) typically improved, relative to PNDP. However, the DI estimations were also impacted by analyte degradation within the sewer system and composite sampler; analyte method recovery from wastewater; and metabolite biotransformation. Although these variables were not corrected for quantitatively, any measured differences between DI and PNDP were largely explained via these phenomena occurring.

The stated PNDP values were also subject to uncertainty. Prescriptions associated with a catchment area may be consumed and/or excreted in a different catchment and drug courses may not be consumed in full. The prescription data, provided by the NHS, is only available in monthly portions. Consequently, PNDP was calculated assuming consistent daily drug consumption, per month. However, short antimicrobial regimens may only be consumed over a couple of days (acute detection in DI), and longer regimens may be consumed across sequential months (under-/over-estimation of PNDP). Due to the size of the catchment and the frequency of prescriptions for the chosen drugs, these errors should have minimal impact on the resultant trends.

Overall, antimicrobials excreted in an unchanged form were often observed to over-estimate DI. This could be attributed to biotransformation, via glucuronide cleavage or direct disposal of unused drug practices. Acetylsulfonamides, TMP, hMTZ, CLR, CIP, OFX, TET, and OTC generally performed well in the estimation of DI, relative to PNDP. The low prevalence of quinolone and trimethoprim metabolites, and the low stability of NIT limited the ability to evaluate these metabolites and their respective CFs.

CFs established in the systematic literature review could not be validated for some metabolites in the case study due to lack of available prescription data (lamivudine, emtricitabine); an inability to quantify biomarkers (nitrofurantoin, doxycycline); being excreted at too low levels (hydroxy-trimethoprim, ofloxacin-N-oxide, desethylene-ciprofloxacin); or insufficient pharmacokinetic literature sources (the nitrofurantoin metabolite, NPAHD).

Consequently, the list of metabolites chosen for DI calculations include aSMX, SPY, aSDZ, TMP, hMTZ, dmCLI, CLR, OFX, CIP, OTC, and TET. Additional data is required to evaluate the correction factors for NIT, NOR, FTC, 3TC, and DOX.

\section{Acknowledgements}

Elizabeth Holton would like to acknowledge University of Bath for funding her PhD. The support of Engineering and Physical Sciences Research Council (EP/P028403/1) is greatly appreciated. The support of Wessex Water Services Ltd and EPSRC Impact Acceleration Account (Project number: EP/R51164X/1, ENTRUST IAA) is greatly appreciated.

\section{Conflicts of interests/Competing interests}

Authors declare no conflict of interests

\section{Credit authorship contribution statement}

Elizabeth Holton: Conceptualisation, Methodology (experimental design, systematic literature review and WBE calculations), Formal analysis, Data curation, Writing - original draft, Writing - review editing; Natalie Sims: Conceptualisation, Methodology (wastewater analysis), Formal analysis, Data curation, Writing - original draft, Writing - review editing; Kishore Jagadeesan: Conceptualisation, Methodology (prescription datasets), Formal analysis, Data curation, Writing - original draft, Writing - review editing; Richard Standerwick: Methodology (sampling, WWTP information); Barbara Kasprzyk-Hordern: Conceptualisation, Methodology (experimental design), Writing-original draft, Writing - review editing, Supervision, Project administration, Funding acquisition, Resources.

\section{References}

1. Department Of Health, U.G., UK Five Year Antimicrobial Resistance Strategy 2013-2018. 2013-18. 
2. Commission, E., Communication from the Commission to the European Parliament and the Council. 2011, Directorate-General for Health \& Consumers.

3. World Health, O., Antimicrobial resistance: global report on surveillance. 2014, Geneva: World Health Organization.

4. Kasprzyk-Hordern, B., Editorial Perspectives: could water fingerprinting help with community-wide health assessment? Environmental Science-Water Research \& Technology, 2019. 5(6): p. 1033-1035.

5. Ort, C., et al., Sewage-based Epidemiology Requires a Truly Transdisciplinary Approach. GAIA - Ecological Perspectives for Science and Society, 2014. 23.

6. Thomas, K., et al., Comparing illicit drug use in 19 European cities through sewage analysis. The Science of the total environment, 2012. 432: p. 432-9.

7. Baz-Lomba, J.A., et al., Comparison of pharmaceutical, illicit drug, alcohol, nicotine and caffeine levels in wastewater with sale, seizure and consumption data for 8 European cities. BMC Public Health, 2016. 16(1): p. 1035.

8. Reid, M., et al., Analysis and Interpretation of Specific Ethanol Metabolites, Ethyl Sulfate, and Ethyl Glucuronide in Sewage Effluent for the Quantitative Measurement of Regional Alcohol Consumption. Alcoholism, clinical and experimental research, 2011. 35: p. 1593-9.

9. Castiglioni, S., et al., A novel approach for monitoring tobacco use in local communities by wastewater analysis. Tobacco Control, 2015. 24(1): p. 38-42.

10. Venhuis, B.J., et al., Dose-to-dose variations with single packages of counterfeit medicines and adulterated dietary supplements as a potential source of false negatives and inaccurate health risk assessments. J Pharm Biomed Anal, 2014. 89: p. 158-65.

11. Causanilles, A., et al., Comparison of phosphodiesterase type $V$ inhibitors use in eight European cities through analysis of urban wastewater. Environment International, 2018. 115: p. 279-284.

12. Ryu, Y., et al., Increased levels of the oxidative stress biomarker 8-iso-prostaglandin F2 $\alpha$ in wastewater associated with tobacco use. Scientific Reports, 2016. 6(1): p. 39055.

13. O'Brien, J.W., et al., Evaluating the stability of three oxidative stress biomarkers under sewer conditions and potential impact for use in wastewater-based epidemiology. Water Research, 2019. 166: p. 115068.

14. Bivins, A., et al., Wastewater-Based Epidemiology: Global Collaborative to Maximize Contributions in the Fight Against COVID-19. Environmental Science \& Technology, 2020. 54(13): p. 7754-7757.

15. Sodre, F.F., et al., WASTEWATER-BASED EPIDEMIOLOGY AS A STRATEGY FOR COMMUNITY MONITORING, MAPPING OF HOTSPOTS AND EARLY WARNING SYSTEMS OF COVID-19. Quimica Nova, 2020. 43(4): p. 515-519.

16. Ahmed, W., et al., First con firmed detection of SARS-CoV-2 in untreated wastewater in Australia: A proof of concept for the wastewater surveillance of COVID-19 in the community. Science of the Total Environment, 2020. 728.

17. Medema, G., et al., Implementation of environmental surveillance for SARS-CoV-2 virus to support public health decisions: Opportunities and challenges. Curr Opin Environ Sci Health, 2020. 17: p. 49-71.

18. Rousis, N.I., et al., Assessment of human exposure to selected pesticides in Norway by wastewater analysis. Sci Total Environ, 2020. 723: p. 138132.

19. O'Brien, J.W., et al., Evaluating the stability of three oxidative stress biomarkers under sewer conditions and potential impact for use in wastewater-based epidemiology. Water Research, 2019. 166.

20. Rousis, N.I., E. Zuccato, and S. Castiglioni, Wastewater-based epidemiology to assess human exposure to pyrethroid pesticides. Environ Int, 2017. 99: p. 213-220.

21. Been, F., et al., Mining the Chemical Information on Urban Wastewater: Monitoring Human Exposure to Phosphorus Flame Retardants and Plasticizers. Environ Sci Technol, 2018. 52(12): p. 6996-7005. 
22. Lopardo, L., et al., Estimation of community-wide exposure to bisphenol A via water fingerprinting. Environ Int, 2019. 125: p. 1-8.

23. Zhang, Y.Z., et al., Wastewater-based epidemiology in Beijing, China: Prevalence of antibiotic use in flu season and association of pharmaceuticals and personal care products with socioeconomic characteristics. Environment International, 2019. 125: p. 152-160.

24. Castrignano, E., et al., Enantiomeric profiling of quinolones and quinolones resistance gene qnrS in European wastewaters. Water Research, 2020. 175.

25. Petrie, B., et al., Critical evaluation of monitoring strategy for the multi-residue determination of 90 chiral and achiral micropollutants in effluent wastewater. Science of The Total Environment, 2017. 579: p. 569-578.

26. Holton, E. and B. Kasprzyk-Hordern, Multiresidue antibiotic-metabolite quantification method using ultra-performance liquid chromatography coupled with tandem mass spectrometry for environmental and public exposure estimation. Analytical and Bioanalytical Chemistry, 2021.

27. Kasprzyk-Hordern, B., et al., Human population as a key driver of biochemical burden in an inter-city system: implications for One Health concept. Journal of Hazardous Materials, 2021: p. 127882.

28. Jagadeesan, K.G., J; Griffin, S; Barden, R; Kasprzyk-Hordern, B, PrAna: An R package to calculate and visualize England NHS primary care prescribing data. BMC Medical Informatics and Decision Making (Under review), 2021.

29. Holton, E.A., E; Fidal, J; Kjeldson, T; Wolfaardt, G; Kasprzyk-Hordern, B, Spatiotemporal urban water profiling for the assessment of environmental and public exposure to antimicrobials (antibiotics, antifungals, and antivirals) in the Eerste River Catchment, South Africa. ChemRxiv, 2021.

30. Lehto, P., K. Kivisto, and P. Neuvonen, The effect of ferrous sulphate on the absorption of norfloxacin, ciprofloxacin and ofloxacin. British Journal of Clinical Pharmacology, 1994. 37(1): p. $82-85$.

31. Frost, R.W., et al., Effects of aluminum hydroxide and calcium carbonate antacids on the bioavailability of ciprofloxacin. Antimicrob Agents Chemother, 1992. 36(4): p. 830-2.

32. Nix, D.E., et al., Effects of aluminum and magnesium antacids and ranitidine on the absorption of ciprofloxacin. Clin Pharmacol Ther, 1989. 46(6): p. 700-5.

33. Ali, A.A., A.S. Gorashi, and Y. Rao, Influence of food on the bioavailability of nitrofurantoin. Indian Journal of Pharmaceutical Sciences, 1988. 50(6): p. 308-311.

34. Ogunbona, F.A. and O.O. Oluwatudimu, Effect of a Non-European (Nigerian) meal on the bioavailability of nitrofurantoin in man. International Journal of Pharmaceutics, 1986. 29(2): p. 191-193.

35. Muchhala, A.T., HP; Kulkarni, RD, Effect of food on the bioavailability of nitrofurantoin products marketed in India. Indian Drugs, 1984. 11: p. 489-94.

36. Hoener, B. and S.E. Patterson, Nitrofurantoin disposition. Clin Pharmacol Ther, 1981. 29(6): p. 808-16.

37. Männistö, P., The effect of crystal size, gastric content and emptying rate on the absorption of nitrofurantoin in healthy human volunteers. Int J Clin Pharmacol Biopharm, 1978. 16(5): p. 223-8.

38. Rosenberg, H.A. and T.R. Bates, The influence of food on nitrofurantoin bioavailability. Clinical Pharmacology \& Therapeutics, 1976. 20(2): p. 227-232.

39. Bates, T.R., J.A. Sequeira, and A.V. Tembo, Effect of food on nitrofurantoin absorption. Clinical Pharmacology \& Therapeutics, 1974. 16(1part1): p. 63-68.

40. EMEA, Committee for veterinary medicinal products: Trimethoprim: Summary report (2). 1997. p. 1-10.

41. Taggart, A.J., B.J. McDermott, and S.D. Roberts, The effect of age and acetylator phenotype on the pharmacokinetics of sulfasalazine in patients with rheumatoid arthritis. Clin Pharmacokinet, 1992. 23(4): p. 311-20. 
42. Vree, T.B., et al., DETERMINATION OF THE ACETYLATOR PHENOTYPE AND PHARMACOKINETICS OF SOME SULFONAMIDES IN MAN. Clinical Pharmacokinetics, 1980. 5(3): p. 274-294.

43. Schröder, H., Deacetylation of acetyl sulphapyridine in man. Journal of Pharmacy and Pharmacology, 1973. 25(7): p. 591-592.

44. Ozaki, T.U., H; Irkuta, T, Studies on metabolism of AM-715 in humans by high-performance liquid chromatography. Chemotherapy (Tokyo), 1981. 29: p. 128-35.

45. Escolà Casas, M., et al., Showcasing the potential of wastewater-based epidemiology to track pharmaceuticals consumption in cities: Comparison against prescription data collected at fine spatial resolution. Environment International, 2021. 150: p. 106404.

46. Verlicchi, P., et al., Comparison of measured and predicted concentrations of selected pharmaceuticals in wastewater and surface water: a case study of a catchment area in the Po Valley (Italy). Sci Total Environ, 2014. 470-471: p. 844-54.

47. Lienert, J., K. Güdel, and B.I. Escher, Screening Method for Ecotoxicological Hazard Assessment of 42 Pharmaceuticals Considering Human Metabolism and Excretory Routes. Environmental Science \& Technology, 2007. 41(12): p. 4471-4478.

48. Khan, S.J. and J.E. Ongerth, Modelling of pharmaceutical residues in Australian sewage by quantities of use and fugacity calculations. Chemosphere, 2004. 54(3): p. 355-367.

49. Coetsier, C.M., et al., Discharge of pharmaceutical products (PPs) through a conventional biological sewage treatment plant: MECs vs PECs? Environment International, 2009. 35(5): p. 787-792.

50. Carballa, M., F. Omil, and J.M. Lema, Comparison of predicted and measured concentrations of selected pharmaceuticals, fragrances and hormones in Spanish sewage. Chemosphere, 2008. 72(8): p. 1118-1123.

51. Khan, S.J. and J.E. Ongerth, Estimation of pharmaceutical residues in primary and secondary sewage sludge based on quantities of use and fugacity modelling. Water Sci Technol, 2002. 46(3): p. 105-13.

52. Thiebault, T., Sulfamethoxazole/Trimethoprim ratio as a new marker in raw wastewaters: $A$ critical review. Science of The Total Environment, 2020. 715: p. 136916.

53. Sun, F.F., Metabolism of Clindamycin II: Urinary Excretion Products of Clindamycin in Rat and Dog. Journal of Pharmaceutical Sciences, 1973. 62(10): p. 1657-1662. 